University of San Diego

Digital USD

1998-02-05

\title{
Ethnographic Inquiry of Social Support throughout Women's Labor and Childbirth Experiences
}

Nancy Jane Pinder Saks DNSc, MSN, RN

University of San Diego

Follow this and additional works at: https://digital.sandiego.edu/dissertations

Part of the Nursing Commons

\section{Digital USD Citation}

Pinder Saks, Nancy Jane DNSc, MSN, RN, "Ethnographic Inquiry of Social Support throughout Women's Labor and Childbirth Experiences" (1998). Dissertations. 281.

https://digital.sandiego.edu/dissertations/281

This Dissertation: Open Access is brought to you for free and open access by the Theses and Dissertations at Digital USD. It has been accepted for inclusion in Dissertations by an authorized administrator of Digital USD. For more information, please contact digital@sandiego.edu. 
UNIVERSITY OF SAN DIEGO

Philip $Y$. Hahn School of Nursing DOCTOR OF NURSING SCIENCE

\author{
ETHNOGRAPHIC INQUIRY OF \\ SOCIAL SUPPORT THROUGHOUT WOMEN'S \\ LABOR AND CHILDBIRTH EXPERIENCES
}

by

Nancy Jane Pinder Saks, MSN, RN

A dissertation presented to the

FACULTY OF THE PHILIP Y. HAHN SCHOOL OF NURSING

UNIVERSITY OF SAN DIEGO

In partial fulfillment of the requirements for the degree

DOCTOR OF NURSING SCIENCE

Eebruary 5, 1998

Dissertation Committee

L. Colette Jones, PhD, RN, FAAN, Chair

Mary Jo Clark, PhD, RN

Susan H. Harris, DNSC, RN 
ETHNOGRAPHIC INQUIRY OF SOCIAL SUPPORT

THROUGHOUT WOMEN'S LABOR AND CHILDBIRTH EXPERIENCE

\begin{abstract}
Nancy Jane Pinder Saks
Dissertation Committee Chair: L. Colette Jones, PhD, RN, EAAN

The purpose of this study was to explore the phenomenon of social support throughout labor and childbirth in order to develop descriptive theory based on the behaviors of the labor and childbirth convoy. Utilizing ethnographic methodology, five women and their support convoys were observed throughout the women's labor and childbirth experience. Word processing and the ETHNOGRAPH software were used for data management, and constant comparative coding methodology was employed for data analysis. Affirmative, affective and aid-giving behaviors were enacted by all convoy members. Consistently, social support was found to be communicated to the laboring woman by her lay supporters by the assurance of continuous presence. Support was provided by convoy members both physically present at the event and available to the woman by phone. The physical environment was seen as a major influence on how social support is transacted. Furniture, lighting and equipment seemed to be the trigger for timing and delivery of support transactions. These patterns require further definition and investigation through continued research. In particular, the body of nursing knowledge can be expanded through validation of these findings with convoy members' description of the temporary nature of role assumption on the part of lay supporters, and description of convoy member activities in different cultures.
\end{abstract}




\section{DEDICATION}

This dissertation is dedicated with much love to my parents

John Charles Pinder and Mary McCracken Pinder my sister

Mary Lou, sister-mother and friend

and to Chip and Clara

for their inspiration of hope for the future and their "disoriented humor" 
I would like to formally recognize my own collegial support network during this process made up of astounding men and women who have all influenced me in their own way. My committee:

Dr. Colette Jones, Dr. Mary Jo Clark, Dr. Susan Harris My colleagues and mentors:

Dr. Marianne Weiss, Dr. June Lowenberg

Dr. Barbara Riegel, Dr. Debra Craig, Ms. Susan

Cummings, Dr. Jane Rapps

My fellow Marion Hubbard scholars:

Dr. Jaynelle Stichler, Dr. June Andrea, Dr. Daniel

Gross

and Mrs. Marion Elizabeth Hubbard for her support of scholarly nursing leadership in health care.

I would also like to acknowledge the labor convoys that allowed my participation in their unique and personal birthing events, all in the name of research to provide optimal care for those who will come to the childbirth setting in the future. 


\section{TABLE OF CONTENTS}

DEDICATION............................

PREFACE..........................

TABLE OF CONTENTS ...................... iv

CHAPTER: $\quad$ PAGE

I. INTRODUCTION TO THE RESEARCH STUDY...... 1

Research Questions.............. 2

Type of study................ 2

Theoretical Perspectives.......... 2

Purpose of the study............ 3

Significance of the Study.......... 3

II. REVIEW OF THE LITERATURE............ 5

Social Support............... 5

Interpersonal transactions..... 6

Convoys................. 9

Labor supports................ 11

Staff nurse............. 12

Nurse-midwife............ 14

Monitrice............... 15

Doula................ 17

Mother................ 19

Male................. 23

Summary................. 25

III. METHODOLOGY OF THE STUDY.......... 26

Epistemologic Assumptions............ 26 
Distinguishing Characteristics......... 28

setting................... 31

Population and Sample........... 32

Data Collection................... 37

Observation................ 38

Peer Review................... 39

Reflection.................. 40

Data Analysis.................. 40

Ethical and Legal Considerations

Informed Consent............. 41

Privacy, Anonymity, and

Confidentiality............... 43

Professional Accountability........ 44

Scientific Adequacy................. 44

Credibility................. 44

Transferability.............. 46

Dependability and Confirmability..... 47

IV. COMMUNICATION OF SOCIAL SUPPORT......... 48

Presencing................... 53

Close Enough to Touch.............. 55

Long Distance Connection............. 56

Routine Maintenance.............. 57

Activities of Questionable Benefit....... 61

$\mathrm{V}$ 
Summary ........................ 64

V. DYADIC AND MULTI-PERSON SUPPORT.......... 66

The Environment................. 66

Dyadic Support

Directors Vs. Actors............ 72

Supportive Activities from the

Laboring woman.............. 74

Supportive Activities from the

Nurse....................... 75

Multi-Person Support

One-to-One Acknowledgment........ 78

Care Conferences.............. 78

Absence of Overt Conflict........ 80

summary.................. 81

VI. CONCLUSIONS AND RECOMMENDATIONS

FOR FURTHER STUDY.................. 83

Implications for Nursing......... 83

Limitations of the study......... 87

Recommendations for Further study.... 89

TABLES

1. Amount of Time Spent Observing.......... 29

2. Ages of Laboring Woman Participants...... 35

3. Convoy Members Present During Observation.. 37

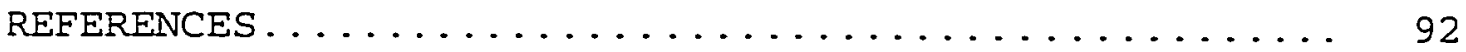

vi 


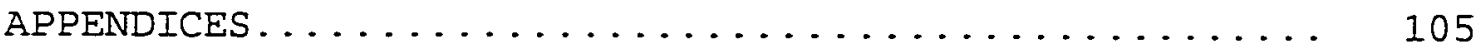

A. University of San Diego Committee

on the Protection of Human Subjects

Approval......................... 105

B. Invitation to Participate............ 106

C. Consent to Participate................ 107

D. Sample Fieldnote Worksheet............ 108

E. Demographic Profile................. 109

vii 
CHAPTER I

INTRODUCTION TO THE RESEARCH STUDY

Consumers have been asking for, and receiving, more family-centered birthing experiences (Shearer, Shiono, \& Rhoads, 1988; Stolte, Myers, \& Owen, 1994; Young, 1992). A family-centered experience includes the presence and participation of one or more other significant individuals identified by the laboring woman as her "supports". This trend has been fostered in part by research demonstrating a positive relationship between social support and desirable childbirth outcomes such as shorter labor and fewer cesarean sections (Kennell, Klaus, McGrath, Robertson \& Hinkley, 1991; Klaus, Kennell, Robertson \& Sosa, 1986).

Little is known about how and why specific social interactions in the form of social support take place between the laboring woman and her support team, including the nurse. Previous studies have focused on the examination of one supporter at a time (Mackey \& Lock, 1989; Sosa, Kennell, Klaus, Robertson, \& Urrutia, 1980). The intent of this study was to generate descriptive theory of the communication of social support in the intrapartum period between and among the attending laymen and professionals. 
Research Questions

The research questions for this study can thus be stated as follows:

1. How is social support communicated during labor and childbirth?

2. What are the patterns of social support among a woman's support convoy when multiple individuals support a woman throughout labor and childbirth?

Type of Study

This study was an ethnographic examination of the phenomenon of social support throughout labor and childbirth. Further, because of the small group membership (fewer than 15 families were examined) this research may be classified as a microethnography (Germain, 1993). The aim of ethnographic inquiry is to uncover the implicit and explicit interactions and meanings of events within the setting in which they most naturally appear (Germain, 1993). The end result of this ethnographic study is a beginning theoretical explanation for the phenomenon of exchanging social support throughout labor and delivery.

Theoretical Perspectives

Steming largely from the domains of sociology and anthropology, ethnography seeks to provide a rich description of the phenomenon under study (Geertz, 1973). The phenomenon is examined and experienced by the researcher from the naturalistic viewpoint of the setting in which it occurs (Schatzman \& Strauss, 1973). This study, which aimed 
to examine the communication of social support throughout labor and childbirth, was done where it "naturally" occurs in the United States, within acute care hospital units. The examination of phenomena within the context where they naturally occur is a basic premise of ethnographic inquiry.

$$
\text { Purpose of the Study }
$$

The purpose of this study was to explore the phenomenon of social support throughout labor and childbirth in order to develop descriptive theory based on the behaviors of the labor and childbirth convoy. This research differs from previous work in the area based on the a priori nature of the research question; that is, it sought a description of the phenomenon and not the testing of any one theory in particular.

\section{Significance of the Study}

Childbirth has been frequently cited in the literature as a pivotal transitional period in adult family-member relationships (Fischer, 1981; Harrison, Neufeld \& Kushner, 1995; Martell, 1990b; Patsdaughter \& Killien, 1990). This study sought to contribute to the body of nursing knowledge by describing the phenomenon of the exchange of social support among those present, including nurses, throughout a woman's labor and childbirth experience. It is thought that this description will assist practicing nurses in a beginning understanding of the phenomenon. Further, it is anticipated that the description provided by this study will assist nurses, and possibly other health care providers, in 
examining their own support behaviors in the childbirth setting. Finally, this description may enable any of the multidisciplinary caregivers accessible to childbearing families to anticipate and plan for their patients' needs. Overview of the Study

With the research questions, theoretical perspective, and significance of the research outlined above, the next two chapters will attempt to further detail the rationale and methodology for this study. Chapter II reviews relevant theoretical and empirical literature on social support which served as a frame of reference for the research questions. Chapter III provides the methodological detail, followed in subsequent chapters by descriptions and interpretation of the themes that evolved from data analysis. 
CHAPTER II

REVIEW OF THE LITERATURE

The purpose of this chapter is to present and discuss some of the body of literature which relates to the research questions. The literature review was completed prior to choosing a research method in an attempt to frame the research questions and to gain direction in defining the appropriate research method. While the reader will see that the empirical literature has most often focused on intrapartum social support from a dyadic perspective, this research proposed a more holistic, family-systems view. The theoretical conceptualization of social support utilized to frame the research questions will be defined and discussed. Further, a review of the literature on intrapartum social support will conclude with rationale for investigating this phenomenon and for the choice of ethnographic methodology. Conceptualizing Social Support

Multiple social support frameworks appear in the literature. Nursing leaders have provided concise summaries of the various conceptualizations and measurement instruments (Lindsey, 1988, \& Norbeck, 1981). While it is acknowledged that there are other social support frameworks, Kahn and Antonucci's (1980) conceptualization of social 
support has been frequently cited in maternal-child nursing literature and provides the closest fit for the kinds of observations suggested by the research questions.

Employing a life-span perspective, these authors posited that, as individuals develop, their needs change over time and they give and receive forms of social support based on immediate circumstances as influenced by past patterns. They defined social support as interpersonal transactions that include one or more of the following: affect, affirmation, and aid. Affective interactions include expressions of love, liking, admiration, or respect. Affirmation transactions are expressions of agreement or acknowledgment of another, and aid is seen as something given to another, as in tangible items, money, information, physical care, and time. The influence of geographic proximity, age, and culture on interpersonal transactions has been discussed in the literature and is summarized below.

\section{Interpersonal transactions}

Geographic proximity. Interpersonal transactions have been examined in a variety of settings including maternity centers and a sampling of relevant literature highlights geographic proximity, age, and cultural patterns as important variables to consider when designing research on the topic. For example, Walker and Thompson (1983) examined the routine aid-giving behaviors of mother-daughter dyads based on their geographic proximity. The study sought to 
determine the association between frequency of contact and aid-giving in age-related pair groups, student women and their mothers and middle-aged women and their mothers. The authors found that, among younger pairs, aid and intimacy were negatively affected by distance. When the pair was physically separated at an inconvenient distance, there was a decrease in the number of some aid-giving behaviors.

Age. Likewise, the age of the childbearing woman has been identified as a factor influencing the type and extent of interpersonal transactions in the childbearing period. For example, primiparous women over the age of thirty-five and pregnant teens have increasingly been perceived as having different needs and concerns from those of other subsectors of women of childbearing age. In one study examining 91 first-time mothers over thirty-five years of age, Reece (1993) examined the women's perspectives on whom they identified as their supporters during pregnancy and in the early postpartum period. The author found that social support was reported most consistently to come from spouse, family, and friends, and was linked positively with the women's self-evaluation of early parenting and lower levels of stress in the postpartum period.

It is not known if Reece's findings of social support from spouse, family, and friends, in a population of women over thirty-five years of age are generalizable to parturient women in all age groups. While health care professionals are beginning to look at the childbearing 
family in differing age groups (Raymond, 1992), age specific social support needs have yet to be clearly defined. Additionally, Reece's study highlights a typical finding in social support studies involving the childbirth period. Other than the spouse, social supporters are lumped under "family" and "friends" and have not been investigated as individuals (e.g., mother, sister, aunt, mother-in-law). Culture. In addition to the variables of geographic proximity and age, culture is recognized as influencing interpersonal transactions related to childbearing. Much has been written about the cultural aspects of childbirth, such as the meaning it holds and the practices surrounding it (Callister, 1995b; Mattson, 1995; Nichols, 1996). It is well established in maternal-child nursing that culture plays an important role in shaping the events of birth and labor (Balin, 1988; Callister, 1995b; Campbell, 1991). However, research specifically examining cultural patterns of interaction between a laboring woman and her supports during childbirth in the united states was not found. While a full examination of culture with respect to social support during parturition is beyond the scope of this paper, one reference stands out as being particularly relevant. In a worldwide survey of cultural patterns, Hedstrom and Newton (1986) looked at one element of interpersonal transactions during childbirth by examining the cultural patterns associated with human touch. The authors indicated there is wide cultural variation on whether touch is used during 
childbirth as affective, affirmative, or aiding. It may be one or a combination of all three and may vary in its duration, provider, and intent throughout the labor and birth. Recognizing that touch, while important, may be viewed as a minuscule component of cultural patterns of behavior, there is considerable work left to be done in investigating other culturally related interpersonal transaction variations in the maternity setting.

\section{Convoys}

Kahn and Antonucci (1980) used the term "convoy" to refer to "the set of persons on whom (an individual) relies for support and those who rely on him or her for support, at any point in time" (p. 269). Convoys are a more transient version of an individual's social support network. Whereas the members of the network are seen as remaining relatively stable throughout the life-span, the convoy is made up of those individuals providing social support during a specific situation or period of life. Women have reported that their social networks in the childbearing period are dominated by their relatives (Cronenwett, 1985a; Chalmers \& Meyer, 1994). In the case of childbirth, women, (the pregnant woman and health care professionals), come together in the maternity setting frequently having never met each other until the onset of labor. The nurses and other health professionals become situational members of the woman's support convoy. Viewed in these terms, family members and friends are but one part of the childbirth support convoy, while 
simultaneously acting as members of the woman's continuing support network.

The notion of mutual support suggested in Kahn and Antonucci's definition of a convoy is applicable to familycentered maternity settings as a woman may choose to have a convoy of supporters present to help and support her during labor and/or delivery. The movement to parenthood, particularly for primiparas, has been conceptualized as a key Iifespan transition (Horman, 1989). From this perspective, the events of labor and delivery occur in the midst of developmental transitions for the woman and her various family members (Horman, 1989, LaRossa, 1983, Mercer, Nichols, \& Doyle, 1988; Murphy, 1990). Social support, as it relates to the childbearing family, has been studied most heavily in the prenatal (Brown, 1986; Giblin, Poland \& Ager, 1990, Norbeck \& Tilden, 1983) and postpartum periods (Cronenwett, 1985a, 1985b). The study reported here relates solely to the intrapartum period for which a comprehensive literature review of the social support convoy is found below.

The convoy a woman may choose to accompany her throughout labor and delivery may include a variety of other men and women, for example, mothers, fathers, sisters, aunts, and cousins. The female non-nurse childbirth supporters may simultaneously be reliving their own previous birthing experiences or, by observation, be learning the birthing role for their own future pregnancies (Saks, 1990). 
However, while much has been written about the motherdaughter relationship, in the childbearing period (Boyd, 1989; Martell, 1990b), no studies were found validating these assumptions of support throughout the labor and delivery period.

\section{Labor Supports}

Although not found studied together as a convoy, a variety of individuals may provide support to the laboring woman during childbirth. Historically in the United States women have provided support to other women during pregnancy and childbirth (King, 1990) with men, other than the male physician, taking on a more active role only in the last three decades. While it is generally recognized that the physician is a part of the delivery team, physicians are usually not present throughout the majority of the labor and delivery period (Rosen, 1991).

other individuals identified in the literature as providing social support during childbirth are labeled "friends" and "relatives" but there is no evidence of research involving them. Up to this point it appears that researchers have chosen to investigate the phenomena through individual analysis of supporters, independent of anyone else in the convoy. Rather than taking a systems perspective, researchers have rationalized choosing the study of one particular source of support, fathers for example, while excluding all others in the setting from the research design. The literature, notably sparse in research 
data in some areas, includes the following as labor supports: nurses (as staff member or midwife), monitrice (self-employed lay women), doulas (lay or trained women, sometimes a nurse, employed by the maternity setting or directly by the laboring woman), mothers (of the laboring women), and significant male others.

Staff nurse as labor support

Research has been done on the supportive behaviors of nurses in the maternity setting. Mackey and Lock (1989) explored multigravid women's expectations of care from the labor and delivery nurse through semi-structured, taped interviews during the last trimester of pregnancy. As one might predict, the authors found that women referred to nurses who had cared for them during previous childbirth experiences when detailing what they expected to occur during their upcoming labor and birth. This finding, in some ways, validates Kahn and Antonucci's theory of social support as building on past experiences.

Two studies were found examining actual supportive activities performed by nurses. McNiven et al. (1992) conducted a work-sampling study on the activities of 18 labor and delivery nurses in a Toronto teaching hospital. The results indicated nurses spent $9.9 \%$ of their time engaged in supportive activities when supportive care was defined as emotional support, physical comfort measures, instructions/information, and advocacy. It is unclear who 
the other members of the women's' convoys were. Since, nursing activities were recorded by the presence of a trained observer, the effect of the observer on the nursepatient interaction is not known.

Using Kahn and Antonucci's social support framework, Kintz (1987) retrospectively surveyed 78 women to delineate nursing support behaviors. The sample consisted of primarily young (mean age 26 years) caucasian women.

This study used the Nursing Support in Labor Questionnaire developed by the study's author. Although a relatively new measure, it is one of the few instruments found that attempts to measure intrapartum social support. The survey includes 20 items which contain descriptions of supportive nursing behaviors. Women were asked to circle "yes" or "no" to indicate whether the nurses who cared for them during a recent childbirth experience had exhibited any of those behaviors. For "yes" responses, the woman is asked to rate their nurses' helpfulness on a 5-point likert scale from (1) not helpful at all to (5) very helpful. Three subscales delineating types of support, (aid, affect, and affirmation) were incorporated.

Cronbach's alpha was measured for the entire 20-item scale (0.93) and for each of the three subscales. The alpha scores were 0.84 for affect, 0.82 for affirmation, and 0.84 for aid. Item scores were added and items ranked in order from the least to the most helpful. When asked in the early 
postpartum period what nurse behaviors they found most helpful during their labor and delivery, the women cited coaching, praising the woman's efforts, providing friendly and personal care, accepting the woman's behavior, treating the woman with respect, and making the woman feel cared about as an individual.

Nurse-midwife as labor support

Nurse-midwives often spend more time with laboring woman than their physician counterparts. Butler, Abrams, Parker, Roberts, and Laros (1993) studied the personal labor support given to patients by certified nurse-midwives. Using a retrospective comparative design, the cesarean section rates of 3551 physician-managed patients and 1056 nurse-midwife-managed patients were analyzed. The women in the study had self-selected a nurse-midwife or physician to attend their childbirth and the effect of this choice on the interaction between the patient and her caregivers is not known. Nurse-midwives were present with their patients for more of labor and delivery than physicians or attending residents. However, no data were available on the types of support provided by either nurse-midwives or physicians/residents. The authors found that patients linked with nurse-midwives experienced fewer cesarean sections, labor abnormalities, and incidents of fetal distress. More documentation regarding the risks and benefits of supportive activities by nurse-midwives would enhance the body of nursing knowledge. 


\section{Monitrice as labor support}

Monitrice is a term used by professional (lay, selfemployed) non-nurse women who privately contract with women to support them throughout childbirth. The use of the term "professional" by lay women is somewhat confusing in the literature where, in the United States, "professional" is most commonly used when speaking of licensed health care providers. The extent of the monitrice-patient relationship varies with some monitrices providing support and education from pregnancy through the postpartum period and others concentrating mainly on supportive behaviors during the labor and birth itself.

In Canada, Hodnett and Osborn (1989b), using an experimental design, on admission to the labor and delivery unit, randomized women to receive either routine nursing care or routine nursing care plus one-to-one support by an experienced monitrice. They found that 103 women cared for by a monitrice remembered receiving more physical comfort measures, emotional support, and advocacy than those women in the control group who had been cared for by staff nurses. The majority of subjects were caucasian middle-class Canadians. Patients cared for by the monitrice used less pain medication and had fewer episiotomies performed. There was little difference in the cesarean section rate between the two groups.

Hodnett and Osborn (1989a) broadened their investigation of social support by studying not only 
physical but psychological outcomes of monitrice-supported childbirth. Social support was conceptualized in four distinct categories: emotional, (encouragement, reassurance), informational, (instructions, explanations), tangible, (physical comfort measures), and advocacy, (acting on one's behalf). In addition to physical data on variables such as pain medication usage, length of labor, and incidence of intrapartum obstetrical interventions, such as the use of oxytocics, forceps, and episiotomy, the authors assessed each woman's sense of being in control. Episiotomy rates and medication usage were lower among those women who had continuous monitrice support. Three variables predicted women's feelings of control during childbirth, (a) expectations of control, (b) the presence of a continuous professional caregiver, and (c) pain medication usage.

Both of these reports from Hodnett and Osborn (1989a; $1989 \mathrm{~b})$ were done with the use of an unnamed survey which categorized nursing behaviors into four groups; physical comfort, emotional support, informational, and advocacy. The dimensions explored were the types of support received from different classes of providers (nurses, monitrices, or mates). Women in the studies recorded which of 20 support items they had received during labor and childbirth and by whom. The number of support behaviors were then averaged to form a score for each of the three provider types involved. No other information on scaling format, reliability and validity, or scoring format was detailed. 
A monitrice is rarely employed by a maternity setting and may be seen as an outsider from the viewpoint of the setting's employees. In some situations this may create conflict between the monitrice and the staff as the monitrice verbally advocates her client's wishes (Shearer, 1984). In these instances, the tension and unrest caused by the conflict may create a more anxious, confused birthing environment, rather than a supportive one.

Doula as labor support

The word 'doula' comes from the Greek terms meaning 'female servant'. Hospitals across the country are seeing a trend toward increased use of doulas based on positive labor outcomes in labors where doulas were present ("Labor of Love," 1997). Doulas, as found in the studies below, and as they are being proposed in the United States, are employees of the birthing facility and have little or no contact with the convoy until the convoy presents at the time of delivery. Focusing directly on the effects of social support provided by a doula, Sosa, Kennell, Klaus, Robertson, and Urrutia (1980) studied the birthing experience of 40 Guatemalan women using a randomized experimental design. Twenty women labored and delivered with the presence of routine medical and nursing staff but no family or friends were permitted to be in attendance. Another 20 women were provided with an untrained lay woman who was continuously present throughout the labor and delivery. The lay labor supports were noted to provide what 
Kahn and Antonucci (1980) would label as affective, affirmative, and aiding transactions in that they conversed, rubbed body parts and held hands, and remained continualiy present and vigilant.

The investigators documented a shorter length of labor in the experimental group ( 8.8 vs. 19.3 hours), and a significantly lower perinatal complication rate. Mothers in the experimental group exhibited more interaction with their newborns shortiy after birth. The authors concluded that even though no prior relationship existed between the laboring woman and lay support, continuous human contact produced beneficial perinatal outcomes. In 1986, using a larger sample of 465 women in the same Guatemalan setting, Klaus, Kennell, Robertson, and Sosa (1986) repeated the study and reported similar findings. Still to be determined is whether continuous human presence affects the outcome or if continuous human presence encourages the maternity staff to attend to the patient more diligently. The cultural differences in social support among Guatemalans versus other cultures warrants attention. It is unlikely that in the United States this study could ever be replicated because the American consumer has demanded family presence in the maternity setting, not restriction of family.

A decade after the initial Guatemalan study, Kennell, Klaus, McGrath, Robertson, and Hinkley (1991), attempted to replicate its findings in a United States hospital. A large sample of 412 healthy nuIliparous women were assigned to 
either a continuous lay supporter (doula) or an observer who was constantly present and recording information but who did not interact with the woman. A control group received no observer or doula in order to test the effect of the presence of the observer as an uninterested supporter. Unlike the Guatemalan studies, family and other supporters were permitted to interact with the women in all three groups for brief periods of time throughout the labor and delivery.

Results of the study indicate a significantly lower cesarean section rate $(p=.004)$ in the doula-supported group (doula 8\%, continuously observed 13\%, control 18\%). The frequency of use of epidural anesthesia, oxytocin usage, maternal fever, and duration of labor was lowest in the doula group, as shown in their previous research. The authors concluded that health professionals should reevaluate current practices in labor support. The results of this study must be confined to comparisons with similar patient populations; that is, low-income Hispanic, black, and white populations.

Mother as labor support

No studies were found where mothers describe their experience of supporting a daughter during childbirth or investigated mothers' support independent of others in the convoy. However, Klass (1988), in a first-person account of supporting her own daughter shed light on types of support that she provided. With her son-in-law actively involved as 
well, she reported having provided verbal praise, warm cloths, conversation, and physical presence. From a personal perspective, her overall appraisal of the experience was positive. No mention was made of any details of her relationship with her daughter either before or after the birth event.

In another account of mothers as childbirth supporters, Stephany (1983), a staff nurse, described her observations of a woman verbalizing to provide support to her daughter. Additional support techniques provided by the mother included continuous presence and tending to hunger and physical and emotional needs. While this piece is anecdotal in nature, it offers practical suggestions for nursing personnel on how to attempt to support mothers who are providing support to their daughters during labor. Suggestions for the nurse include "teaching, listening, and offering...physical presence" (p. 345). These may be valid and useful suggestions for the nurse but their efficacy has not been tested.

Marteli (1990a) employed a written qualitative tool to survey 64 primigravida daughters and 60 of their mothers. All of the delivering daughters responding to the study were either married or in a stable relationship with a male partner. The average age of daughters was 24 years. The average age of mothers in this all-Caucasian sample was 49 years. A questionnaire asking these pregnant women and their mothers about their contact and aid patterns with each 
other and about their feelings of closeness was administered by mail. Content analysis of transcripts revealed three emerging themes. First, there was some degree of change in the relationship during the pregnancy reported by both mothers and daughters, particularly the resolution of some past conflict. Second, the type of aid provided by the mother to the daughter, (i.e. physical and/or emotional), was important to the dyad although geographic distance played some role in how aid was exchanged. Lastly, the dyad commented on how the roles of other individuals, such as the daughter's husband, influenced the mother-daughter relationship. These findings, while supporting that geographic distance is an important variable, reinforce the need to examine interpersonal transactions between dyads within the convoy as well as among the convoy as a whole. Other authors have argued against including the mother as doula or continuous support person to her daughter during childbirth. In their book "Mothering the Mother", while supporting the idea of continuous female companionship throughout labor and delivery, Klaus, Kennell, and Klaus (1993) stated the following five reasons for not advocating the mother as the ideal birth companion. First, mothers lack experience with the technology, terms, and physiology involved in labor and delivery. Second, many of the "mothers" at this point in time had their own children in the 1950's and 1960's and they may not have been delivered under "ideal" circumstances. Third, there is often 
geographic distance between mother and daughter in today's society. Fourth, there is danger that the mother may usurp the position of the father of the baby. Fifth, there may be psychologic distance between the mother and daughter.

To date, the validity of these arguments has yet to be studied. While it is commonly accepted that most lay individuals do not possess the technical and physiologic knowledge comparable to that of a health care professional. it is not clear to what degree this kind of informational support in itself influences laboring women and their childbirth outcomes. Regarding Klaus et al.'s (1993) concern that mothers may not have delivered under "ideal" circumstances in the 1950's and 1960's, it is difficult to define an "ideal" birthing situation. This reasoning seems groundless since in the past century many aspects of childbirth have changed such as setting, instrumentation, and medication and as a society Americans continue to refine their idea of the "ideal" birth. The effects on the daughter's childbirth experience of a mother who simultaneously is reliving her own childbirth experience has yet to be examined and associated with either negative and/or positive effects.

Geographic distances between Eamily members and their expectant relatives, as mentioned previously, deserves consideration. If a family member is unable to be physically present during the labor and delivery, for travel or any other reason, perhaps that would be an excellent time to 
explore the use of a doula. Emotional distancing may be of more concern. Regardless of geographic distance, existing conflict between family members and the expectant woman, or among any of the convoy members, would create additional challenges in the maternity setting. Male as labor support

Nurses in the labor and delivery setting recognize a woman's male companion as their "patient" too, including husbands, boyfriends, fathers, brothers, uncles and others. Unlike any other supports in the setting, the male who presents as the woman's "significant other" often comes to the childbirth experience with a history of a physically intimate relationship with the laboring woman not experienced by the other convoy members (Callister, 1995a). Coaching the male with suggestions on the timing of and techniques for providing comfort are standard nursing practice (Malestic, 1990). However, not alI male supports report feeling comfortable in their role as primary labor supporter (Nichols, 1993). Berry (1988), in a descriptive, retrospective study of 40 new fathers, found that men experienced increasing stress as the labor progressed, a finding corroborated by Nichols (1993). Additionally, 858 of Berry's subjects did not expect to feel the way they felt or reported that they were unable to be as helpful to the woman as they had imagined they would be during the prenatal period.

Berry's findings (1988) that men did not actively do 
what they had previously envisioned may be reflective of the roles that male supports play during labor, as outlined by Chapman (1992). Using grounded theory methodology the researcher interviewed 20 couples four weeks after delivery. Three roles emerged from the descriptions the men gave depicting the behaviors they engaged in during labor and delivery to support their partner: coach, teammate, and witness. Interestingly, the majority of men enacted the witness role, physically separating from the bedside and becoming more of a passive observer than an active participant. Providing additional evidence of physical separation Bertsch et al. (1990), in an observational study of 14 births, found that fathers' physical distance from the laboring woman increased as labor progressed. The testing of these roles, coach, teammate, and witness, among other partner populations as well as with others who are present in the support role may provide insight into the kinds of support women receive in parturition. Nurses and childbirth educators, together with the expectant couples, may need to rethink what is expected of males as supports.

In summary, no studies were found which examined the interactions between the individuals supporting a woman in labor and their collective interactions with the laboring woman. In theory and practice, many questions remain unanswered relevant to these interactions. By having knowledge of how support roles are assigned, enacted, and negotiated within the support convoy, nurses then may be 
able to define their own roles more clearly.

Summary

Kahn and Antonucci's conceptualization of social support as a mutual, multi-person phenomenon, which fits well with the childbirth setting, served as a frame of reference for the research questions posed. With the exception of Bertsch et al. (1990) the review of the empirical body of literature on intrapartum social support indicates that, to this point, an a priori stance, such as that employed by this ethnographic methodology, has not been taken in nursing research investigating intrapartum social support. The instruments developed and used in the studies cited here lack a clearly defined description and theory of the nature of intrapartum social support. There is also dissension as to whether nursing support should be labeled social support. 
CHAPTER III

METHODOLOGY OF THE STUDY

The intent of this chapter is to outline the method utilized in conducting the research and the related epistemologic assumptions. Also included are details of application of the method in the field in seeking to answer the research questions. Ethnographic inquiry was chosen because of its applicability to the research questions which sought to ascertain the nature of intrapartum social support. This study may also be labeled as qualitative family research because of its focus on "experiences within families as well as between families and outside systems" (Gilgun, 1992, p. 24).

Epistemologic Assumptions

Ethnography is a research methodology which has its' roots in sociology and anthropology (Lowenberg, 1993). It is a qualitative approach that has been defined by Leininger 1985, p. 35) as "the systematic process of observing, detailing, describing, documenting, and analyzing the lifeways or particular patterns of a culture (or subculture) in order to grasp the lifeways or patterns of the people in their familiar environment". In this study, the labor and delivery unit was considered the environment familiar to the 26 
majority of women in the United states as the place where birthing occurs.

A major belief underlying ethnography is that "people's behavior can be understood only in context" (Boyle, 1994, p. 162). Actions by humans cannot be analyzed independent of their natural setting. To do so might distort the meaning or relevance of the behavior. For example, Iying fullyclothed on the floor of someone else's home in the midale of the night, with the lights on, may be considered unusual in some settings. But when an expectant father proceeded to do this in the labor, delivery, recovery room of the acute care maternity center, it was perfectly acceptable and, despite his fatigue, he was praised for his endurance and physical presence for the laboring woman. In ethnography, it is not enough then, to solely describe human action. The ethnographer seeks an understanding of "why the behavior takes place and under what circumstances" (Boyle, 1994, p. 162).

Another epistemologic underpinning of ethnography lies in its attention to culture. Culture, defined by Germain (1993, p. 237) is the "learned social behavior or the way of life of a particular group of people". Cultures (and subcultures) can be identified in all types of organizations, institutions, and settings by characteristics such as norms, beliefs, values, and social structure. It is within these cultural norms that individuals function in society. Ethnography, through in-depth, personal experience 
in the setting, allows the researcher to observe the characteristics of the culture. This study focused on caucasian woman in Southern California experiencing childbirth where the majority of woman currently deliver in an acute care maternity center.

Distinguishing Characteristics of Ethnography Many variations of qualitative research methodologies exist in the Iiterature. Boyle (1994) outlines four characteristics of the ethnographic method that, when established, assist in distinguishing it from other qualitative research methodologies. The first is the holistic, contextual nature of the method. The researcher goes out in the field or natural setting where the phenomenon under investigation is thought to occur. Prolonged, intensive contact with the people in the setting, while labor intensive, is expected to yield the context and meaning of phenomena. I observed five labor support convoys in two different facilities during a total time of 59.5 hours as shown in Table 1 . 
Table 1

Amount of Time (in Hours) Spent Observing

Convoy \# Hours spent in observation

1

2

3

4

5
6

17.5 (over 2 admissions)

9

14.5

12.5

The second distinguishing characteristic is reflexivity. The ethnographic researcher cannot entirely distance him/herself from the setting nor can he/she be entirely neutral to the observations. Reflexivity "implies that the researcher is a part of the world that she or he studies and is affected by it (Boyle, 1994, p. 165). The researcher's rational knowledge gained from past experiences, knowledge from authoritative figures and intuitive knowledge blend together with the activities of the setting allowing the researcher to draw inference and obtain understanding (Munhall, 1993).

Having worked and taught extensively in the maternalchild field, including the labor and delivery area, I was quite familiar with the routine activities of the setting. 
Adding to my own knowledge and comfort level, I had previously worked a considerable amount of time in the two facilities where the observations took place.

Prior to initiating the study, my role as a participant observer was defined with the supervisory nursing staff at each facility. They verbalized understanding that I would not be providing any hands-on care and that, should the occasion arise where I identify a medical emergency, I would seek out the staff rather than independentIy initiating treatment. During the observation periods, there were no incidents of medical emergency where I felt that I needed to intervene.

My role as participant observer was also described to each member of each convoy. Each was asked to try to ignore the fact that I was present and try to forget that I was there. However, in each of the five convoys reflexivity was evident in that one or all of the members attempted to include me in such things as holding the baby and partaking in food and breaks from the room. I answered briefly, to be polite, trying then to reinforce my need to stay noninteractive with non-verbal behaviors such as diverted eye contact.

Attention to emics and etics is the third distinguishing characteristic of ethnography. The emic perspective refers to the "insider's view, or the informant's perspective of reality" (Boyle, 1994, p. 166). The etic perspective refers to the "outsider's" view or the 
"researcher's abstractions, or scientific explanation of reality" (Boyle, 1994, p. 166). Both views need to be employed during the research process to produce comprehensive interpretation in the form of concepts and theories. It is this mix of emic and etic perspectives as a part of triangulating data collection which allows the researcher to draw a unique picture of the phenomenon. The researcher can choose to present the data in the written report from one perspective rather than another, emic or etic, clearly stating the epistemologic approach taken (Boyle, 1994). While both the emic and the etic perspectives were used in data collection during the study, and analysis, the findings are presented here primarily in the etic perspective, attempting to describe the observed behaviors. The production of ethnography is the fourth distinguishing characteristic. Boyle (1994) stated that the product of ethnographic research can take a variety of forms dependent upon what phenomenon is studied and the experience of the researcher while investigating. Regardless, the strength of the study's conclusion, particularly as it relates to nursing and other health professions, is in the reporting of "pragmatic outcomes for both theory and practice" (Boyle, 1994, p. 169).

Setting

A large, integrated health care system in Southern California known for its high yearly delivery volume was used as the setting for the study. This site was chosen 
because it was thought to have an environment which would allow this researcher to obtain the richest data possible (Lofland \& Lofland, 1984).

In these facilities, labor, delivery and at least the first hour of recovery, unless complicated, take place in the same room which is often referred to as an LDR. The labor and delivery units have an open visitation policy meaning that family and friends can enter and leave the LDR area as they wish, regardless of time of day. However, the units reserve the right to restrict visitors based on the desires of the patient and/or observed clinical need. AlI observations for this study took place on the LDR unit. I chose not to travel into the operating room with one woman who delivered by cesearean section with only one supporter in attendance.

Population and Sample

Approval to conduct the study was obtained from the University of San Diego Committee on the Protection of Human Subjects (Appendix A) and the health care facilities' Institutional Review Board. The two health care facilities, part of a larger integrated health care system, are governed by a common Institutional Review Board and therefore the approval granted covered both facilities used for the study. Following receipt of approval, participants were recruited. A purposive sampling technique was utilized. In this sampling method study participants and events are sought because of the richness of the data that can be obtained 
using ethnographic methods (Sandelowski, 1995). Potential participants were contacted through prenatal education classes at a busy local hospital and recruitment from prenatal care settings. The inclusion criteria were primiparous, Caucasian, English-speaking women, planning to have more than one supporter present throughout labor and delivery, and willing to allow my presence for observation and recording of field notes. After a verbal overview of the project from the researcher, a brief questionnaire was completed by interested participants (Appendix B). The questionnaire assisted in the identification of age, ethnic background and the woman's plan for individuals to support her during labor.

The initial plan to recruit subjects from the prenatal education classes yielded only one convoy. Multiple negative factors precluded enrollment of convoys from the prenatal classes. The classes are usually completed around the 32nd week of pregnancy. Near term at 40-42 weeks, several couples had forgotten they agreed to notify me on their admission to labor and delivery and were near or past delivery when we made contact. For three consented couples I was out of the state on the day that they delivered and therefore unable to observe them.

To increase the chance of successful observations, after revision of the research protocol through both the University of San Diego Committee on the Protection of Human Subjects and the facilities' Institutional Review Board, 
participants were recruited by flyer and verbal contact at physician offices and antenatal testing centers, yielding the final five convoys.

Because birth experiences may be strongly influenced by cultural practices, variation in the ethnicity of the woman was minimized as a demographic variation by purposive sampling (Sandelowski, 1995). Additionally, Sawyer et al. (1995) argued that qualitative investigators should attend to phenomena related to their own knowledge of culture and their own sensitivity to culture to produce more culturally competent findings. For these reasons, because I am caucasian, only caucasian women were enrolled and no attempt will be made to generalize the findings from this ethnography (Sandelowski, 1995). The cultural makeup of prenatal class attendees varied by geographic location. At some prenatal class sites all class participants were Hispanic and therefore could not be recruited.

Caucasian women over the age of eighteen who indicated their intent to have multiple individuals present during labor and delivery were approached. After explaining to each individual the expectations of study participants, potential subjects were asked to sign consent forms agreeing to study participation (Appendix C). Labor convoys were sought until it appeared that no new data was emerging. The ages of the laboring women participants, spanning the parturient years, were from 19 to 41 (see Table 2). 
Table 2

Frequency of Ages of Laboring Women Participants

\# of laboring women Age range (years)

1

$19-22$

1

$27-30$

2

$35-38$

1

$39-42$

All of the pregnant woman had attended prenatal classes with at least one member of the convoy. None of the convoys attended prenatal classes as a group. This may in part be because childbirth education instructors currently are discouraging women from bringing more than one support with them to class because of space constraints. Classes need to be held in rooms with ample floor space to allow women to recline for relaxation exercises.

When the women, each with one supporter, are in the room, there is often little space left for anyone else. Another factor was geographic distance. Each convoy had a different configuration of members (see Table 3 ) and some of the convoy members lived out-of-state and came to the setting just around the time of delivery, expecting to return to their own homes afterward. 
OnIy the third convoy had the same nurse throughout the entire observational period. The other four convoys experienced two or more nurses as labor progressed through multiple scheduled unit shifts. None of the births involved a monitrice or midwife. Midwives are not currently involved in care at the two facilities where the observations took place. Physicians made brief (under 5 minute) visits to the participants' I.DR and spent, on the average, less than an hour in the room delivering the newborn when summoned to the LDR by the nurse.

One out of the five labor observations ended with cesearean delivery. All were considered low risk at the outstart of labor and had epidurals placed some time during labor, after first trialing breathing techniques. Pitocin was used in four of the cases and no overt complications were experienced by any of the women or their infants. With the sample size of five convoys, each with a different mix of individual supporters, although the lack of delivery complications support Kennell, et al.'s (1991) findings that companionship during labor reduced complications, it would be premature to conclude from this study that companionship was the reason for the lack of complications seen here. 
Table 3

Convoy Members Present During Observation

\begin{tabular}{|c|c|c|c|c|c|}
\hline \multirow[b]{2}{*}{ Participant } & \multirow[b]{2}{*}{1} & \multicolumn{3}{|c|}{ Convoy \# } & \multirow[b]{2}{*}{5} \\
\hline & & 2 & 3 & 4 & \\
\hline Laboring woman & $\mathrm{x}$ & $\mathrm{X}$ & $\mathrm{x}$ & $\mathrm{X}$ & $\mathrm{X}$ \\
\hline Woman's husband & & $\mathrm{X}$ & $\mathrm{X}$ & $\mathrm{x}$ & $\mathrm{X}$ \\
\hline Woman's sister & & $\mathrm{x}$ & 2 & & \\
\hline Woman's mother & $\mathrm{x}$ & $\mathrm{x}$ & & & \\
\hline Woman's father & $\mathrm{X}$ & $\mathrm{x}$ & & & \\
\hline Woman's mother-in-law & & $\mathrm{x}$ & & & \\
\hline Woman's sister-in-law & & & & $\mathrm{X}$ & \\
\hline Woman's female friend & $\mathrm{X}$ & & & $\mathrm{X}$ & \\
\hline Doula & & & & & $\mathrm{x}$ \\
\hline Nurse & 2 & 5 & $x$ & 2 & 2 \\
\hline
\end{tabular}

Note. $\mathrm{x}=1$

Data Collection

Triangulation of methods was employed to obtain study data in an attempt to capture the most complete picture of the nature of intrapartum social support (Germain, 1993). This was accomplished through the use of qualitative data sources including observations, peer review of coding, and personal reflection (Boyle, 1994). 
Observation. Participants were asked to contact me as they and their supports were admitted to the hospital for labor and delivery. I then joined them at the hospital labor room and remained with them through the labor. For three convoys, observations extended one to two hours following the birth. For one convoy, after deciding on the need for a cesarean section, the physician declined to allow more than one supporter in the operating arena. The observational period ended when the convoy was last all together, in the operating preparation area. For the other convoy, the female labor support left two hours prior to delivery, thus ending the convoy interaction and field notation. The time spent in the setting during the labor and birth events with each woman and her convoy ranged from 6 hours to $171 / 2$ hours. Observation of the verbal and nonverbal interactions between all of the primary participants in the setting was done for the intrapartum phase. Observations of the participants was limited to what took place within the labor and delivery room, excluding such places as hallways and waiting areas.

Strengths of this participant observation method of data collection include having the researcher in the event setting among the informants, the opportunity to collect large amounts of detailed information, and the ability of the researcher to capture both verbal and nonverbal communications (Marshall \& Rossman, 1989). Weaknesses of this method include the potential for misinterpretation of 
informant communication by the researcher, the inability to replicate events, the potential for ethical dilemmas involving the researcher, and its' high dependence on the cooperation of the informants (Marshall \& Rossman, 1989). One father-to-be, during my first few minutes in the room, related to his wife that he felt "caged". I took this to mean that he was sensitive to my presence and obvious notetaking. Once later, during a brief lull in activity in the room, he said directly to me, "I'm still not used to you being here yet". However, each time when given the opportunity to discontinue the convoy's participation in the study, he declined.

were recorded in a discrete manner, either by stepping out of the room for brief periods or while sitting in the room (Appendix D). This logging of events, is congruent with the ethnographic methodology because it reinforces the "receptive, almost passive" nature of data collection (Lofland \& Lofland, 1995).

Field notes were transcribed into word processing format for use in the ETHNOGRAPH computer program for data management and analysis purposes. The served as the primary source of data for the study. Additionally, a brief demographic survey was completed by each study participant prior to labor and delivery (Appendix E).

Peer review. A doctorally prepared nurse colleague with an extensive background in labor and delivery nursing was engaged to review the first sets of concurrently, but 
independently of the researcher. This additional analytic input yielded ongoing feedback and support to the process of coding and categorizing, validating my own coding and categorizing. On comparison, there was a high rate of congruence between our two sets of codes. It was an integral part of narrowing the focus of observation until the point of saturation.

Reflection. My thoughts and feelings were recorded in writing on the observational field notes throughout the labor and birth event. In addition, summative were completed after each labor and birth experience which included notations on researcher introspection. Where possible, thoughts and feelings from the observations were verified with the study participants. For example, I took "bathroom breaks" when other convoy members did so, allowing them to approach me about the study. AII did and I was able to get additional impressions from them regarding how they felt the labor experience was progressing for themselves and the laboring woman. Reflection continued as subsequent observations yielded additional data. Discussions with nurse colleagues, presentation of preliminary findings at a nursing research symposium, and the subsequent comments of the nurse attendees provided further reflection.

Data Analysis

The logged words and actions recorded in the field notes during observations served as the primary source of data (Lofland \& Lofland, 1995). The technique of constant 
comparison was applied to the logged data once it had been entered into the ETHNOGRAPH. This began immediately after the first observation and was done within 3 days of each subsequent observation.

After initial coding in the Ethnograph, I opted to complete coding and categorizing by hand because it seemed more time efficient and accessible. In accordance with constant comparative methodology, data analysis was an ongoing process of coding and narrowing the focus of subsequent observations in the field. Constant comparative methodology was chosen as the data analysis method because of its flexibility of options for handling the detail of the field notes and because its" aim is the "generation of theoretical constructs that ....form a theory" (Hutchinson, 1993, p. 2001.

In accordance with the chosen methodology, data from the field notes were coded by units of meaning. Similar codes were then grouped into categories that seemed to most realistically occur based on the data. Categories of data were compared to one another and where relevant, categories were clustered (Germain, 1993).

Ethical and Legal Considerations

Informed consent. Prior to the observations, the pregnant woman and her primary lay supports had the study verbally explained to them, including the potential risks and benefits and voluntary nature of participation, after which they were invited to participate. Many of the hospital 
unit's nursing staff had the nature of the study explained to them in a very general way at a staff meeting approximately one month prior to the first expected delivery dates for the participants. At that point, nurses were offered the consent form to sign in an effort to ease staffing restrictions which potentially could have arisen during the labor and delivery observation if nurses chose not to participate.

It was planned that if no nurse on duty was willing to participate at the time of any observational period, that woman and her lay supporters' participation would be endangered and the researcher would consider deletion of that woman from the study. On the contrary, during the study all nurses encountered were gracious, interested participants. At the time of the observations several nurses not in attendance at the staff meetings had not previously been briefed about the study. In those cases, I stepped out of the room and gave an explanation to each one, prior to obtaining their consent. Additionally, all of the nurses assigned to the study convoys were encouraged to hear that their participation in the research would assist them as staff nurses in achieving their expected annual performance goals.

Once the patient consented to participate, the primary physician or physician on duty was contacted and the study was explained to him/her in a general way to alert them of my presence. Additionally, information sessions were given 
to the Neonatal Resuscitation Team which includes

physicians, nurses, and respiratory therapists. They

received a general explanation of the purpose of the study. However, there was no plan to obtain individual consent from any of those staff members because their presence at the birth, if at all, is primarily in support of the neonate and not the mother or her Iay supporters and therefore, while relevant, was not considered a part of the central focus of the observation. During the study, the Neonatal

Resuscitation team was called to only one of the deliveries after meconium was noted on pushing.

Privacy, anonymity and confidentiality. The privacy of each individual was maintained by the coding of field notes for later identification. Only the researcher and peer reviewers knew how the coding system was established and notes to that effect were kept in a separate location from the field notes. Transcribed field notes did not have any identifying information on them except code numbers. All confidential study information was kept in a locked file cabinet to which only the researcher had access. Examples from field notes included here are without identifying descriptors.

Study subjects were discouraged from relating any secrets to the researcher that they did not want included in the report (Munhall, 1988). On one occasion, a nurse pulled me aside to get my personal opinion about a laboring woman's behavior. Following a gentle reminder to the nurse that the 
role of the researcher did not allow interaction of that sort, the "consult" ended. At one other point, I was very uncomfortable hearing of a probate transaction where the financial details, including individuals' names, were discussed. Initials only, and a general description of the content of conversation were recorded on the at that point. Professional accountability. Because I am a licensed nurse, I negotiated my role as a professional with the labor and delivery administrative staff prior to entry. The agreed upon plan called for me to immediately report any danger to mother or her infant to the appropriate staff for their attention. No direct patient care was expected or delivered from the researcher. Upon my arrival on the LDR unit for each observation, I checked in with the charge nurse, insuring that she was clear on my purpose and intent. Scientific Adequacy

Trustworthiness or rigor in the conduction and analysis of this research were monitored in terms of standards suggested by Lincoln and Guba (1986). The authors identify credibility, transferability, dependability, and confirmability as criteria for evaluating qualitative research.

Credibility

Prolonged engagement and persistent observation. Lincoln and Guba (1986, p. 77) defined prolonged engagement as "lengthy and intensive contact with the phenomena in the field". The authors defined persistent observation as "in- 
depth pursuit of those elements found to be especially salient". Consideration was given to the length of time spent in intensive contact with the setting, 59.5 hours, to insure adequate exposure to obtain the transactional data necessary to address the research questions. Length of contact was assessed on an ongoing basis to insure adequate observations of social support transactions and collection of data until saturation and to address the research questions of types and patterns of social support. For consistency, I was the only observer.

However, due to the extended length of each observation, researcher fatigue became a problem. The accuracy of after several intense hours of observation and recording must be noted as possibly influencing the quality of the data. The "visitor" chair was very uncomfortable to sit in after more than a half hour and, following the first observation, I brought small pillows for the sides and back of the chair which increased my comfort, and therefore my ability to concentrate on observation.

After having worked a full routine workday, one observation of $121 / 2$ hours extended into the middle of the night and, after the participant had an epidural placed, she and her husband rested in a completely darkened room. Fatigue and the urge to sleep were fought with caffeine, walking in the hallway and change of chair position. It was resolved by the observation period ending, but the last hour of from that participant were reviewed with the fatigue 
factor in mind.

Triangulation. Otherwise known as cross-checking (Lincoln and Guba,1986), triangulation involves the comparison of data from a variety of sources. This is thought to enhance the content validity of the research (Germain, 1993). Where possible, study participants were asked for their thoughts and feelings regarding not only their own experience, but the perceptions of the other individuals present, including the researcher. The female support in the fourth convoy was a nurse familiar with the setting. She provided intermittent feedback and her interpretation of events during breaks outside of the room. Additionally, whenever available, photographs, records or other potential sources of information were utilized.

Peer debriefing. A nurse with a critical care background and not involved with the external audit process was engaged to support the researcher both emotionally and intellectually. This nurse openly discussed the progression of this research in content and/or process on an ongoing basis for the length of the study. Lincoln and Guba (1986, p. 77) see this process of "exposing oneself to a disinterested professional peer" as a way of providing support and self-monitoring for the researcher. Transferability

The criterion of transferability as described by Lincoln and Guba (1986, p. 77), involves the accumulation of "thick descriptive data" in the form of "narrative developed 
about the context so that judgements about the degree of fit or similarity may be made by others". This criterion is addressed in the final presentation of the research findings to follow which includes a synopsis of the emerged categories and/or themes. Where applicable, each is illustrated using actual descriptions derived from the field notes.

Dependability and confirmability

Conducting an external inquiry audit assists qualitative researchers in establishing the dependability and confirmability of their findings (Lincoln \& Guba, 1986). To complete this process, the field notes were given to an impartial auditor for a review of the management and analysis of the fieldnote data. A doctorally prepared nurse familiar with ethnographic methodology and constant comparative methodology acted as this auditor. Additionally, utilization of the constant comparative methodology assisted in creating the necessary audit trail of progress during the coding and categorizing of data. 
CHAPTER IV

\section{COMMUNICATION OF SOCIAL SUPPORT}

The first research question sought description regarding how social support is communicated during labor and childbirth. Each convoy member, including the laboring woman, both gave and received social support. As circumstances of labor changed from minute to minute, the forms of social support seemed to change. Supporting Kahn and Antonucci's (1980) categories of social support behaviors, affective, affirmative and aid-giving behaviors were all seen. Common supportive behaviors in each domain are discussed below. They are broken into two categories of behaviors, those behaviors toward the laboring woman and those behaviors among the convoy, because there appeared to be distinct patterns of transactions. There were behaviors exhibited toward the laboring woman that were not seen enacted toward anyone else. For example, gentle kissing and patting on the head were seen toward the laboring woman, but never from one member of the convoy to another.

Affective behaviors toward the laboring woman commonly took the form of light kissing on the face, hands or shoulder, gentle touching of the shoulders, legs, and top of the head, and verbal admiration such as "I'm proud of you." 
Affective behaviors among the other convoy members were seen in hugging, wrapping arms around another's waist or shoulders, and verbal respect such as heard when one hourold Grandmother asked her husband, "Grandpa, do you want to hold her (the new baby)?"

Affirmative behaviors were enacted to the woman in labor by way of repeated questions ascertaining present pain status, such as, "Where does it hurt?" and "Are you hurting again?". There was also acknowledgement that the laboring woman was leading their behavior and in need of their support in that the supporters attentively complied with even the smallest of indications that their assistance might be needed. For example, on several occasions women on the bed moved their feet around, and in doing so, reached for the sheet to avoid getting their legs tangled. Seeing this, supporters often sprang into action, even getting up from their chairs, to help move the sheet. This extreme level of attention to the laboring woman from all members of the convoy characterized all labors.

Convoy members affirmed the presence of their fellow supports through a lot of head nodding, and verbal dialogue to establish a plan of action to address the laboring woman's current needs. They also acknowledged each other's presence and activity through incremental self-monitoring. For example, one female supporter, pausing to take in the current activity in the room, noticed that all three supporters who were present were standing up around the bed. 
"Look at us, all standing at attention", she said. While pointing out their presence and willingness to assist, it also forced each to evaluate their own behavior.

Aid-giving behaviors toward the laboring woman often took the form of verbal encouragement. One father, giving practical advice, told his daughter, "Thing is you have to relax. Just loosen up in between your contractions." Also, there were frequent provisions of comfort through such things as bed and furniture adjustments, massage of body parts, food and drink, and assistance with the medical equipment, particularly the intravenous tubing and fetal monitoring leads.

Aid-giving behaviors among the convoy were seen in seemingly simple gestures. For example, one nurse, speaking to a participant's husband, asked him if he wanted a bag of ice to rub on his wife's sore back and a stool to sit on while he rubbed his wife's back. In another group the doula monitored the compact disc player, keeping it running and assessing with the laboring woman if the volume was satisfactory. Convoy members frequently shared food, drink, and chairs and made sure that they were not physically in each other's way.

These demonstrations of aid, affirmation and affection reinforce the findings of Tarkka and Paunonen (1996) in their study of social support during childbirth. The aim of their study was to determine what networks of support were available to laboring women and to link the social support 
provided from the network to the mother's experiences during childbirth. The authors used Norbeck's Social Support Questionnaire, a well-tested general social support tool and a scale they designed based on Kahn's theory of social support. That 20 item, 5-point Likert measure consisted of 7 questions on aid, 7 questions on affirmation, and 6 questions on affect. Mothers in the study reported that members of their childbirth support network included their spouses, parents, siblings, other relatives, Eriends, colleagues, neighbors, and health care professionals (most often the public health nurse familiar to them through their prenatal experience).

Conducted in Finland, the research indicated that family members provide much of the support to laboring women. Also, while all three types of social support, aid, affirmation, and affection, were provided by the midwives, the majority of the emotional support for women in labor was provided by the midwife. However, in that setting the role of the midwife is enacted as the United States' equivalency of a cross between a labor and delivery nurse and a physician's assistant. Comparison is therefore difficult with variation in the roles.

In each convoy there appeared to be one "main" supporter of the laboring woman who remained most vigilant at the bedside and seemed to get a larger proportion of social support from the other convoy members, as opposed to giving support. It was not universally the woman's husband 
who was the "main" supporter nor was it ever the nurse. In this study three husbands, a mother, and a sister appeared to be the main supporters, spending more time at the bedside and in direct physical contact with the laboring woman than any of the other convoy members, including the nurses. In the case where the mother served as the main supporter, there was no husband or boyfriend present and none were scheduled to be there. However, in the case where the laboring woman's sister served as the main supporter, the husband was present throughout most of the event. It would be interesting to investigate whether this "support the main supporter" behavior was preestablished in any way through discussion in the prenatal period.

Supporting the main supporter was enacted in various ways. The main supporter seemed to be expected to spend the most concentrated time at the woman's side. Therefore many of the support activities provided by the other convoy members involved providing food, drink, blankets, clothes and comfortable seating to the main support. Additionally, when a comfort measure was suggested to ease the pain of upcoming contractions, the necessary props were forwarded to the main support for him or her to employ. There may in some ways be a hierarchy of support in place among the convoy with the woman garnering the most support and the main supporter getting an amount of support less than the laboring woman but greater than that given to other convoy members . 
While no profoundly new types or classifications of social support were identified, specific patterns of support emerged as being dominant modes used during the labor and childbirth event. For example, providing the laboring woman with the constant physical presence of one of the lay supporters was evident in each of the observed convoys. Presencing

This physical "being there" or presencing on the part of the lay supporters was demonstrated in each of the convoys and may, in part, account for the lack of complications experienced by the convoys. Kennell et al. (1991) found that even when they placed a nonparticipating observer in the room with laboring women, there was a lower cesarean section rate and incidence of complications such as maternal fever as opposed to women who labored alone. They posited that factors such as fear, which may contribute to pain perception, may be alleviated simply by the presence of another human being in the room.

Assuring that a lay supporter was always present in the room with the laboring woman was negotiated among the lay convoy members in a multitude of ways. In one instance, after two sisters were present in the LDR for about a half hour and the husband had been present since early morning the husband said, "I'm gonna leave. You sisters are having your sister talk." Discussion followed where the sisters advised the husband of what preparatory tasks he should accomplish while he was at home, such as showering, getting 
more film, and bringing baby clothes. The sisters stayed in the room until he returned.

Presence did not mean sitting idly in the LDR. Supporters filled their time with a variety of activities including reading the daily paper, playing cards, watching television, working on laptop computers, and making business phone calls.

Bond, Keen-Payne \& Lucy, (1995), in a study of women relinquishing their infants for adoption after laboring and delivering without any lay supporters, found in postpartum interviews that the women clearly wanted continuing nursing presence. Other studies have confirmed that laboring women value the presence of the professional nurse caregiver (Bryanton, Fraser-Davey \& Sullivan, 1994; Collins, 1986; \& Mackey \& Stepans, 1994). Some are calling for the nurse (called midwife in the UK) to be the individual who provides the continuous support in the form of her presence as a means of meeting the changing needs of the laboring woman (Anderson, 1996). From the data I collected here, the lay supporters fill the need for continuous presence and the nurses' views of the need for continuous presence are not known. The nurses working with the study convoys did not need to consider arranging for continuous physical presence for the woman because the support convoy consistently saw to it that one of them was present. Further investigation could assist in delineating whether laboring women have a preference, or further still, report greater satisfaction 
and better outcomes, with the continuous presence of lay versus professional personnel (Hodnett, 1996).

Close enough to touch. Almost as a subset of presencing was the tendency for the person present to remain within touching distance of the laboring woman. This was accomplished by the supporters in a variety of ways including sitting on the bed, standing by the bed, pulling a chair next to the bed or standing at the foot of the bed. Repeatedly this positioning of self was in some way followed by touching the laboring woman, most commonly on the arm or gently stroking her leg. A mother, whispering conversation to the laboring woman and her husband, without permission, took the hairband out of the laboring woman's hair, rubbed and brushed the hair to fluff it up and rebanded it. A sister, settling in to read a Cosmopolitan magazine, pulled her chair up until the chair and her legs were touching the bedside. The male supports were not noted to move away from the bed as labor progressed as had been found by Bertsch et al.(1990). Instead, they maintained their contact, sometimes getting even closer, forehead to forehead, as labor progressed.

The negotiation of who was positioned by the bedside was done seamlessly without verbal exchange. The bed that the laboring woman was in logistically only allowed so many individuals to be within touching distance at any one time. Lay supporters, when near the maternal-fetal monitoring systems, repeatedly gave up their bedside position without 
comment when the nurse came into the room. When the nursing personnel left the room, the convoy members resumed their positions close to or on the bed.

However, on one occasion a woman's husband was standing directly beside the laboring woman trying to hold her leg while she attempted pushing. The woman's sister came up behind the husband and quietly instructed him on how to hold the leg. The nurse unexpectedly stepped over and said, "I'm going to trade places with you here" (to the husband, indicating that she would hold the leg). The husband did not move and gently but firmly replied, "I was going to stand here". The exchange ended there.

Long Distance "Connection"

Each labor convoy in its own way kept the laboring woman in contact with friends and/or family outside of the hospital setting. In one scenario, the woman's friend appeared to take it upon herself every hour or so to call a few designated persons and update them on the latest happenings. In another scenario, the woman's mother played the role of telephone operator. She screened calls and placed calls. Additionally, two of the laboring women made several calls to friends to give their own updates. It was noted that after providing the update to the female on the other end of the line, the laboring woman did a lot of listening.

It would be most interesting to further explore the kinds of support, perhaps in the form of advice and 
encouragement, that these long distance supporters provided. While it is obvious that geographic proximity limits the possible types of aid-giving behaviors enactable, use of the telephone allows for social exchange during labor and childbirth. Future technologies, such as videophones, if installed in childbirti settings, may enhance the types of social support possible.

Routine Maintenance

Observations of all of the convoys revealed the members seeking to maintain normalcy of daily routine for themselves, and at times, even for the laboring woman. Mealtimes were adhered to with the exception of when they coincided with the delivery event itself. This seemed to be monitored by all members of the support convoy who, noting the time of day, ascertained the hunger status of other support members, including the nurses and laboring woman. Communication with friends, family and colleagues was also maintained. Pagers were left on and functioning, creating beeping and buzzing noises at what sometimes felt like awkward moments of intense contraction pain for the laboring woman. For example,

Patient: Eyes closed, head back on chair.

Husband: In between contractions, coaching sister on how to use camera.

BEEPING NOISE

Husband: "Check to see if that (pager) is mine." 
Dad: Up to see if it is his or son-in-Iaw's.

Mom: On the phone to leave a message to her workplace on how they can get a hold of her. "I can't believe how much I just got done here".

Husband: Quietly describing rise of contraction on monitor to wife.

Sister: Blowing nose.

Mother-in-law, Mom, Sister: Discussing Girl Scout cookies.

At the extreme of maintaining normalcy for the laboring woman, one woman who was a partner in business with her mother, urged her mother to continue a phone conversation with a business contact because the conversation provided the laboring woman with the necessary distraction to negotiate a uterine contraction. In other instances, the pursuit of normalcy seemed to logically clash with the activity in the room. The sister of one woman, snacking on cough drops, mistakenly allowed them to spill onto the Eloor during a tense moment of seemingly painful contraction negotiation. Repeatedly, lay supporters brought food with strong aromas such as specialty coffee and onion-laden deli sandwiches into the LDR room and ate it because it was around their own "normal" eating time. This continued sometimes even in spite of verbal recognition that odors were making the laboring woman nauseous and, in some cases, had led to emesis. One female friend ordered a Big Mac by phone while the laboring woman vomited. Later when the 
sandwich was delivered she ate the highly aromatic sandwich in the room while the patient experienced another round of emesis and was helped by her mother. Still later, as the woman ended another emesis period, the friend held the emesis basis for her with one hand, glanced over her own shoulder at the bedside table and inquired to the woman as to whether or not she, the supporter, could have a piece of the cookie that the woman's aunt had delivered!

At other times, routine or familiar situations were used by convoy members to help them assimilate what was happening and enhance their ability to participate in a meaningful way. In verbally coaching the laboring woman for example, one woman's father, in urging her to maintain pushing behaviors in the second stage of labor, said, "That's a girl...just like doing sit-ups." Earlier that same gentleman had held the attention of everyone in the room for about a minute's time by relating his own hospital experience with his dying father. He described how he and his siblings had filled a bedpan full of ice and treated their dad to beer and pizza in his hospital room 17 years ago. He likened the party atmosphere to what he was then experiencing with his daughter's first childbirth experience and made the connection that the activities in the room were tailored to the "guest of honor", that being the patient in the bed.

One husband and wife, separate from the other supporters present, used past experiences familiar to them 
as a way to assist the wife through contractions. Their brief "trips" were all similar in structure, but the detail varied. Several "trips" are detailed below.

Patient: As contraction begins. "Where are we going?"

(Cue that she was feeling a contraction).

Husband: Glancing at monitor. "We're going to Mammoth. We're in the car with our skis on the roof. It is very dark outside and there are a lot of stars in the sky. We're driving fast."

At a later contraction:

Patient: Closed eyes (cue) as contraction begins.

Husband: Glancing at monitor. "We were sailing in the bay. We were coming through the channel and it was tight in there. There were birds flying around that we have never seen before. We had to do lots of quick tacks. It was a beautiful day."

And still later:

Patient: Puts head back, eyes closed. (Cue)

Husband: "We're at Mammoth now. There is so much snow.

It is so quiet and peaceful. When we wake up

there was so much snow. We had to dig out."

These retold memories of past events, which were mutually experienced by the laboring woman and her support, seemed to provide distraction and focus for the woman and a personal way for the husband to provide support. 


\section{Activities of Questionable Benefit}

Some observed behaviors particularly deserve attention in future investigations to determine how they were perceived by the laboring woman and other convoy members. As the observer-as-participant, The three behavior categories are identified as annoying and/or non-supportive, recounting personal stories, and nursing support.

As some behaviors were enacted, they seemed to me to be annoying and possibly non-supportive. For example, at times some of the lay members turned up the volume on the television or compact disc player to a level where everyone in the room had to somewhat raise their voices to be heard. Food bags were crumbled and chairs were dragged noisily across the floor of the room at the height of contractions, with the laboring woman concentrating hard on mastering them. On other occasions, women who had been laboring extensively were drawn into the birth of other newborns on the unit. Convoy members, sticking their head out of the labor room door, would call everyone's attention to a newborn crying nearby and stop there as if to say, "That woman did it already". A more supportive response, such as, "That will happen in here soon" was not a part of the transactions.

In another example, a female friend stood by the uterine activity monitoring printer to watch for signs that a contraction was beginning. She called out a warning as the contraction began and then talked through the whole 
contraction. Several times in a row she would say, "It's ALMOST peaking, it's ALMOST peaking". The laboring woman in that case, after experiencing three or four contractions while listening to "It's ALMOST peaking, it's ALMOST peaking" gently suggested to her friend that it, "Might be better to just wait and let me know when it's coming down." It was clear that the convoy members felt that their role was to "do" something. Whether or not the participant's activities in the room were "supportive" (as against nonsupportive) of the laboring and delivery process is not known from these observations.

In three of the five convoys some of the lay women present were of childbearing age and had recently (aIl within 8 years) delivered a child themselves. Unsolicited, they intermittently verbally called to mind the events of their personal labor and delivery experience, relating what happened to them in comparison to what was then taking place during the woman's labor. These "recent mothers" also gave detail of what was to come, seemingly in a way to assist the laboring woman in concretely preparing herself for imminent events.

For example, when after rupture of membranes had occurred, one laboring woman said:

Laboring Woman: "Oh, I feel it dripping" (amniotic fluid)

Friend: Acknowledged that that feels "odd" and then 
related the mess she had made when she had to use a bedpan during labor.

Another sister to the laboring woman shared her experiences frequently, matching her experience with events currently taking place. When her sister completed a contraction and declared, "I felt that one in my hips", the woman, appearing to acknowledge the previously unexperienced kind of body pain involved in labor, said, "I just remember like an outof-body experience".

There were no outright signs noted indicating that the laboring woman had perceived the sharing of the convoy member's personal story as non-supportive. The women seemed to listen intently, commenting very little, but also not engaging in extensive questioning which may have elicited more discussion. It is not known what purpose this recalling of birthing events serves for the story's teller, nor for the laboring woman as the receiver. It would be interesting to pursue in more detail why these women felt the need to engage in those transactions and benefits received.

Nurses did not come and stay in the room continuously during peak times where I perceived the laboring women were in need of their support. One example of that was when the intravenous pain medications were not sufficient and anesthesia had been called for epidural insertion. It also appeared that during the pushing stages of labor the women were not consistently attended by the nurse and, as an observer, this absence seemed non-supportive. Lay convoy 
members used what techniques they knew to support women through those moments and there was no expression of disappointment or anger at the nurse for not being there and providing additional suggestions for comfort measures or not stepping in and personally comforting the woman.

These behaviors did not elicit any one kind of response from any of the convoy members. There did not seem to be any consistent recognition among the convoy that these behaviors might be non-supportive. It is assumed that convoy members were not as acutely aware of each transaction as I was as the observer. Follow-up validation is needed to gain an understanding of whether or not the behaviors were noted by the convoy members, and if so, how they were perceived. Summary

The lay members of multiple-support labor convoys appear to take on ownership of the responsibility for providing continuous human contact for the laboring woman. With few exceptions, lay supporters remained vigilant at a distance from which they could easily make physical contact and did so often. While these physically present laymen were identified as the woman's immediate supports, the data revealed a pattern of seeking/receiving support from others outside the immediate childbirth setting. All of the members of the childbirth convoy attempted to maintain some level of normal routine despite the uniqueness of the events of labor and childbirth. Occasionally their behaviors appeared to be of questionable benefit to the laboring woman. Validation of 
these observations with both the lay and professional convoy members may further bring to light the motivation for these support and coping mechanisms employed in the setting. 


\section{CHAPTER V \\ DYADIC AND MULTI-PERSON SUPPORT}

The second research question sought a description of patterns of social support among a woman's support convoy when multiple individuals support a woman throughout labor and childbirth. The data revealed two major categories regarding the patterns of support exchanged; dyadic and multi-person support. Each of these major categories was overshadowed by the effect that the physical environment appeared to have on when and how social support was exchanged.

The Environment

The data from this study revealed that the physical environment of the labor, delivery and recovery room served as a major stimulus for behaviors from all of the convoy members. The data provide interesting insights into how the architecture, design and furnishing of the LDR room plays a part in human behavioral transactions during the childbirth event.

Westreich, et al. (1991) in a study of the influence of birth setting on the father's behavior toward his partner and infant found statistically significantly more coaching 
and verbal encouragement from the father in the traditional birth setting compared to others in a birthing room. In this study of the acute care setting, I found that without any verbal exchange or instruction, when the nurse arrived in the room, she headed directly to the maternal-fetal monitoring side of the bed. Simultaneously the lay supporters would rise from that side of the bed, unless they were sitting on the bed, and would move to somewhere at the back of the room or foot of the bed. Some nurses attempted to thwart the laymen's behavior by repeating on several trips into the room, "You don't need to get up. You are fine there." Those words of reassurance to the lay supporters went mostly unheeded and the supporters continued to rise when professional personnel arrived.

The lay convoy members did not seem annoyed by their self-established need to repeatedly remove themselves from a space in the room that, while close, could adequately hold two individuals. In other cultures perhaps the close physical proximity of the laymen with the nurse in that corner of the room would not be as much a concern. In addition, this behavior was reinforced by the laboring women who did not protest when their supports moved to a further distance, even mid-contraction.

The physical distance between the convoy members and the nurse is conceptualized as "social" space by Purkis (1996) who posited that nurses establish their identity in 
the social encounters with patients through the physical spacing they maintain. Purkis debated what "therapeutic" spacing is or might be in nurse-patient environments and suggested that positioning of self is a proactive behavior which portrays meaning and therefore influences the responsive behaviors of others. This explanation may account for the behaviors of the convoy members in the labor and delivery settings. If well-established nurses give behavioral cues to those in the setting as to expected workspace, those cues may, in turn, be influencing the movement of the convoy.

Further, lay convoy members assumed the role of furniture movers without direction. Particularly when the room was being set up for epidural insertion or delivery, the lay supporters were not passive observers. They rose from their places quickly and after taking nonverbal cues from the professional caregivers, moved any and all furniture to another part of the room out of the way of the main activity.

For the nurse, assessment of the environment seemed to be integrated into other activities. On no occasion was a nurse noted to enter the room, pause to assess the activity in the room, and then proceed. If the nurses were assessing the environment and activities in the room, they did so while simultaneously or after assessing the physiologic parameter monitors. Some nurses, after establishing the physiologic stability of both mother and baby, then turned 
toward the labor convoy and stood looking around at the whole room, appearing to "assess" the convoy's needs. While directed most often to the laboring woman, the nurse's assessment also included asking about environmental concerns such as the amount of light, chairs and heat in the room. This same type of take-it-all-in observation was occasionally enacted by some of the women convoy members as well. When demonstrated by the convoy women, it was usually followed by some kind of environmental adjustment such as turning the television on or off, adjusting the lights brighter or lower, changing the compact disc, or clearing away trash from table surfaces. Environmental control seemed to be the responsibility of the convoy and not an issue that the laboring women concerned themselves with. Validation of this environmental assessment and control with the nurses and convoy members in a post-delivery interview would be helpful in gathering information on what, if anything, the nurse looks for environmentally upon each return to the $L D R$ room and in support of the activities and welfare of the convoy.

Research on the influence of the environment in the health care setting has focused heavily on the patient and health care staff (Bouchard, 1993; Cooper \& Hasselkus, 1992; Namazi \& Johnson, 1996). The physical environment is acknowledged as playing a major role in individuals' stress levels and well-being (Gulrajani, 1995; Heerwagen, Heubach, Montgomery \& Weimer, 1995; Williams, 1989). In the case of 
the labor and delivery setting each convoy member is a part of the environment, and therefore affected by it.

Baldus (1989) stated that health care settings whose physical plants reflect medical intervention encourage dependent behavior by the patient and controlling behavior by the health care personnel. The labor and delivery units used for this study all have been renovated within the last five years. In response to consumer desires, the rooms are furnished to present a more home-like atmosphere, as opposed to a medical one. The floors are hardwood, the furniture, including beside table and headwall, are done in oak. Floral valences top off homey looking vertical blinds. Medical equipment is cleverly hidden behind oak and there is an amoire with television, compact disc player and video cassette player. From insider knowledge I know that patient satisfaction has risen since the renovations. However, it has not been established whether or not the environmental enhancements toward a more home-like atmosphere have fuIly met patients' and their supporter's expectations and needs. Personally I found that the visitor chairs provided little comfort when used for even short periods of time. The discomfort was distracting to my data gathering efforts. Convoy members sitting in the same type chairs over extended periods did not voice discomfort, but were noted to be very mobile, getting up and down frequently. It is not known whether the architecture of the chairs, or any other part of the setting, specifically altered interpersonal transactions 
among the convoy. However, members of the convoy were restricted in their movement in the room based on the preestablished architectural layout.

Hutton and Richardson (1995) presented a theoretical model of the concepts involved in health care environments by use of the term "atmospherics." Atmospherics are those parts of the environment that are physical and can be controlled in some way. In their model, furniture would be considered an atmospheric. In theory, individuals have a perception of what certain settings will be like and once in the setting, their behavior is altered by their response to the atmosphere they actually encounter in the setting. One's behavior towards others is then, in part, a result of response to interaction with the environment.

This theory may, in part, explain the interpersonal transactions seen in the labor and delivery setting. It is difficult to determine the effect of preconceived expectations of the settings from the field work done here. There is ample evidence that the environmental changes experienced hour by hour were linked with behavioral responses. For example, when the obstetrical technician came in to the room to set out the sterile tables, she was quite elaborate in her gestures in setting up the sterile fields and equipment. Once finished, she gave instructions to everyone in the room as to how the newly draped areas should be avoided. The convoy members appeared to listen to instructions carefully. They then quickly modified other 
parts of the room to accommodate this new change, and still be a functioning support. They moved chairs, personal equipment and curtains, but not medical equipment or patient-related equipment such as the bedside table or the laboring woman's bed.

All laboring women had attended prenatal classes, part of which includes a tour of the rooms. All of the convoys brought supplies which required access to some of the homelike equipment in the room, such as compact discs. Likewise, the childbirth education curriculum includes suggestions for pain control and distraction which include the use of music. In that way, convoy members, at least those who attended class or had previously experienced childbirth in a similar setting, knew what to expect. It could be theorized that the behaviors seen in this and other regards were the result of preconceived "education and training" as to what proper labor support and behavior entails. It would be most interesting to pursue a pre-delivery interview with convoy members which included questions eliciting what kinds of behavior each expects to enact, with probing into where they think they might have acquired that notion.

Dyadic Support

Directors vs. actors. The laboring women were at times observed to be very directive in telling others how to care for them and what roles some of the lay convoy members should play. Some examples are as follows: 
"Get some sandwich or something while you are out."

"Stop by Kim's place to fill her in."

"You guys can turn on the TV if you want."

"Why don't you move around on this side so I can see your face?"

(To friend who is verbally describing what she sees on the fetal monitor for each contraction). "You might wait on the monitor part until you see it coming down. It scares me, I don't know when you say it's going up."

There was little, if any, hesitancy noted from the supporters following a directive from the laboring woman. One woman, after negotiating a difficult contraction, noted that everyone in the room had stopped their activity, creating silence.

Laboring woman: "What happened? You guys stopped talking"?

Woman's Eather: "We were waiting. That (contraction) was a biggie."

Laboring woman: "Keep talking, it distracts me." Following this instruction, on the next few contractions three of the lay supporters seemed to pointedly carry on a conversation while watching the progression of the uterine contraction print out on the monitor. They readily followed the woman's command.

The laboring women seemed less inclined to direct the actions of the nurse but, regardless, incidents of that kind 
occurred. For example, following the change of shift from one nurse to another, when the new nurse arrived in the room the laboring woman pronounced to the nurse, "I need help with pushing". In another instance the laboring woman directed the doctor by saying, "P. (the father of the baby) wants to cut the cord."

Supportive activities from the laboring woman. Although most professional maternity nurses would probably anecdotally acknowledge support from the laboring woman to other members in the convoy without having to do a research study, there is poor documentation of such in the literature. Laboring women showed repeated concern for the physical comfort and nutritional needs of those around them. During a rest period from pushing, one woman said to her husband, "Are you okay? Need a break? Am I lying on your foot?"

Such expressions of concern for another during critical times such as pushing seemed to be made possible with the infusion of epidural medication. When not in pain, the women seemed to look outside themselves to monitor the activity in their immediate surroundings. Prior to epidural insertion however, a pattern of irritable, curt responses which dramatically changed the tone of all interactions in the room, was noted from the laboring woman to her supporters. When a husband reached over to assist his wife in readjusting the uterine monitor belt, the woman snapped, "No. Don't", and swatted at his hand. 
When not in severe pain, women also showed concern and support for individuals not immediately present. One participant worried about her out-of-state mother who lives alone and wondered how she was handing waiting for news of the delivery. She resolved her concern by having her female friend make a phone call and update the mother on labor progress.

Supportive activities from the nurse. When Kintz (1987) asked postpartal women what nurse behaviors they found to be most helpful, the women cited coaching, praising the woman's efforts, providing friendly and personal care, accepting the woman's behavior, treating the woman with respect, and making the woman feel cared for as an individual. With the exception of coaching, the nurses observed in this ethnographic study demonstrated all of the other behaviors as those noted in Kintz's study. Malestic (1990) stated that providing suggestions for support to the male labor support was a standard nursing action. In this populuation however the lay supporters took on the majority of the coaching to the laboring woman, and to each other. While the nurses did demonstrate those behaviors, the frequency of those supportive behaviors was greater from the lay supporters than from the nursing staff.

The nurses observed in this study seemed to have what might be called a "room monitor" approach to interacting with the laboring woman and her family. They came into the room at the time of their scheduled mandatory assessment, 
often announcing to those in the room "it is time to chart again." Most seemed prepared to step in for a crisis, but none occurred. They notified the physician of patient needs not within their purview and called the physician to the room for delivery. The majority of interventions for comfort management suggested to the laboring woman emanated from the lay, and in one case professional, convoy members. It has been suggested that our modern practices in childbirth have detracted somewhat from women's experiences with childbirth and the meaning that it holds. Treatments and procedures performed by medical and nursing personnel may in fact be socializing those caregivers to focus on safe, competent care rather than a more holistic approach which includes providing meaning and life-long, psychological perspective to the event (Waldenstrom, 1996).

In essence, the nurses observed in this study did not function as a part of the support convoy, but apart from it. In some respects they may be viewed as a "part of the environment, something else for the convoy members to react to." How these nurses perceive themselves in relation to the convoy is not known and would perhaps shed light on their somewhat separatist behavior. Additionally, it may be noted that the IDR setting, by its nature, is family health nursing. Nurses lacking adequate formal preparation in how to work with families may not be comfortable and/or knowledgeable about the skills and interventions required. 
It is interesting to note that this observation of somewhat separatist behavior on the part of the nurse fits with a recently published view of nurses' enactment of social support. Unlike the conceptualization of the nurse as a "member" of the support convoy in this study, Hupcey and Morse (1997) might conceptualize the role of the labor and delivery nurse as being outside of the labor convoy as they make a distinction between social support and professional support. After completing a comprehensive review of social support literature, the authors posited that a prior personal relationship is critical to the exchange of social support and thus is inherently different from the professional support provided by health care workers who are often establishing a relationship as they care for the patient. They feel a key component to social support is reciprocity and that relationships with care providers do not provide the same level of reciprocity, with patients gaining more than caregivers. Obligation to other, the duration and expectations of the relationship, trust and the type of service obtained determine whether support is seen as professional or social.

Evidence for this separation between professional and social support in the childbirth setting may be supplied by Tarkka and Paunonen (1996) who did not find nurses listed as supporters when they asked postpartum women who their supports had been during the childbirth process. 
Multi-person Support

One-to-one acknowledgment. A team-like atmosphere was noted in each of the support convoys. One nurse complimented her convoy team by saying, "This is the easiest I've had to work all night. You guys are great". Other comments such as "We work well together", "We're doing good", and "We're making progress", reflected a spirit of teamwork and pride in group accomplishment. Yet, simultaneously, it was repeatedly reinforced that the beneficiary of the outcome of the team's effort would be the laboring woman who would have "a beautiful baby."

Participation on the part of the support convoy included activities of acknowledgment. The supporters acknowledged each other by taking food breaks together, attending to each other's comfort needs regarding such things as seating and room temperature and asking each other information about their home and work Iives. These impromptu behaviors seemed to aid in building a sense of team.

Care conferences. Two categories of conferencing were observed. The first seemed to focus on strategic planning and the second on evaluation of the professional caregivers. Regarding strategic planning, seemingly at critical points in the labor lay supporters were noted to "conference" at the foot of the bed or back of the room and discuss what actions should be taken next. They spoke in hushed tones but well within earshot of the laboring woman and the lay supporter who remained in physical contact with her. These 
strategic sessions seemed to involve brainstorming about options for pain management, in particular, looking for interventions that they themselves could explore. The result of the session was often agreement on a pain management strategy that was then passed on for the lay supporter in physical contact with the laboring woman to try.

The second type of care conferencing observed surrounded evaluating the professional caregivers. At least once during each childbirth experience observed, when the nurse left the room, one of the lay supporters initiated a verbal critiquing session that started with an analysis of the current nurse and then moved to the doctor and other nursing personnel if any others had been involved. The critique included assessment of their opinions of the nurse's friendliness, professional behavior, and the value they placed on her professional judgment. The convoy appeared to be convincing themselves of their opinion. After a discussion of a patient's fluid status in relation to her gestational diabetes, the husband reaffirmed the nurse's competence with the rest of the convoy by summarizing, "She clearly knew what she was talking about,". The laboring women were observed intently listening to the views and observations of the convoy members, occasionally adding their own views. While no conclusion was explicitly drawn, it appeared that at some level the convoy members had established a level of consensus regarding the credibility of the nurse provider. 
Nurses should be apprised of the factors that support convoys are considering when they conduct these impromptu evaluations and be made aware of the effect, if any, that these evaluations have on the nurse working as a member of the convoy for the rest of the childbirth experience. Follow-up focus groups, with the nurses as participants may be one way in which nursing staff can gain more insight into how their behavior is perceived by laboring women and their convoys. Ahmann (1994) emphasized that in order to work successfully in providing family-centered care, nurses need to move into a more collaborative care model that recognizes and accepts diversity in family coping styles and caregiving behavior.

Absence of overt conflict. It is interesting to note that no incidents of outright conflict between dyads or among the convoy was observed. It is, however, my experience as an instructor of nursing in the labor and delivery arena, that expressions of anger and aggression do occur. Additionally, Klaus et al. (1993), fearing that a mother's behavior toward her daughter in labor would usurp some of the role of the father of the baby and cause conflict, suggested that mothers as supportive companions would be problematic. No evidence of conflict, specifically between the mother and any other member of the convoy, was detected. In this study's Iimited number of birthing events, the convoys generally worked well together and did not appear to be "holding back" anything because of my presence. 
Nonetheless, my presence should be considered as a possible deterrent to a show of conflict. It also may be that women who felt they had a cohesive convoy, with no fear of revealing inter-group conflict, self-selected for participation.

Summary

Two major patterns of interpersonal transactions were revealed, those among dyads and those exchanged among multiple members of the convoy. The physical and social environment, in part fostered by the nursing staff, appears to play a large role in the exchange of social support behaviors in the intrapartum period. The small sample and lack of multicultural data collection minimize the ability to generalize the findings. Yet, the themes delineated here provide the basis for future research questions to explore.

In theory then, components imbedded in the social support of a laboring woman appear to include constant presencing, physical touch, and long-distance conection. Supporters work to maintain a balance between routine activities and an acutely heightened sense of attention to the needs of the laboring woman.

As a group, being a part of a support convoy involves working within the environment in a balance between planning and enacting activities and following the directions of others, while receiving support oneself. An unvoiced sense of team purpose and goal draws the convoy members together to strategize effective behaviors. Kahn and Antonucci's 
(1980) conceptualization of mutual support among convoy members was seen demonstrated by all of the convoy members.

Social support during the whole childbearing period continues to be studied with the focus on linking some exchange of social support with positive birth, parenting, and relational outcomes. Since no previous research was found that sought to delineate social support behaviors in the intrapartal period from an a priori approach as the one undertaken in this study, the findings of this study make a unique contribution to the literature. Where previous inquiries into social support in the intrapartal period have looked at support on an individual transaction basis, the data from this study reveals group behavior in an effort to assist the laboring woman. Where instruments employed in previous studies have been constructed from such theoretical Erames as Kahn and Antonucci's social support (i.e. Kintz, 1987 ) or self-constructed by the individual researcher to address specific research questions (i.e. Hodnett \& Osborn, 1989a), validations of this preliminary theory of multiperson support in the childbirth setting may lead to, and heighten the need for, an instrument designed to measure the components of convoy support as described through this study. 
CHAPTER VI

CONCLUSIONS AND RECOMMENDATIONS FOR FURTHER STUDY Implications for Nursing

Very little research literature exists describing in detail social support transactions occurring in the childbirth setting. There are a number of methodological issues surrounding the implementation of such investigations, all of which create a formidable task for nurse researchers. Also, population-specific variables such as ethnicity and age (De Anda \& Becerra, 1984) add to the challenge of generating overarching theory addressing social support among a labor and childbirth convoy. However, the description of behavioral transactions as found in this study are offered to nurses in the setting as a way of gaining more insight into the behavioral patterns of the participants. Additionally, if follow-up research indicates that the nurse provides a limited amount of certain kinds of support, then the profession should not be taken by surprise by a call from consumers for additional supports such as doulas and monitrices.

The labor and delivery environment worldwide, like other patient care settings, is seeing multiple pilot 
projects exploring different mixes of types of caregiver by academic preparation and function. It is not practical to attempt to generalize the findings of this study to any other setting, especially in light of the fact that delivery settings vary widely as to the roles which nurses, midwives, physician's assistants, doulas, and monitrices are assigned. In the face of cost-cutting measures, if registered nurses in the United States are to remain at the bedside in the LDR rooms, further evaluations are needed to identify the nurse's role as a contributing member of the support convoy. Already some nurses are speaking out regarding strained resources in labor and delivery settings and are calling for doula supports to provide uninterrupted emotional and physical support for laboring women (Lothian, 1994). This study was conducted under the philosophical belief that the nurse is a part of the social support convoy. In light of Hupcey and Morse's (1997) argument that nurses cannot be a part of the social support network because of the nature of their behaviors and the length of their relationships with patients, it will be important for nurses in the future to establish their expected support behaviors in the labor and delivery setting and then to share those expectations with consumers.

This study then, which provides some descriptive insight into the behaviors of convoy members, conveys to the nursing profession a preliminary view of events in the setting. Regardless of what skill mix arrangements or 
convoy makeup appear in particular labor and delivery settings, the information gained from the observations of this study contributes to what nurses now know about how convoys are enacting their support of laboring women. With further investigation into these patterns, nurses might be able to create flexible roles, varying the kinds and amounts of social support that they provide from one woman to another, based on the setting and convoy makeup. For example, if relating events of one's own labor and delivery experience is a supportive component provided by the convoy, then nurses caring for women who have no "experienced mothers" as a part of their convoy may consider moving away from their tradition of not interjecting details of their own experiences and move toward incorporating that sharing of personal detail as a connected, supportive part of the role.

The educational preparation of the nurses and their perceptions of how realistically they function in the social support role is information needed prior to the implementation of any practice change. Communication transactions among members of the support convoy are complex and family systems theory, communication theory, and theories of life-span perspectives might enhance the basic education of nursing students, particularly in the obstetrical arena, so that explorations of practice can be undertaken. 
In terms of convoy education, ongoing dialogue with those lay and nursing personnel providing childbirth education is essential. For example, the concept of "team" is not currently a formal part of childbirth education curricula. As the role of the individual supports during the labor experience is further delineated, that information should be utilized to shape the content and approach to preparing women and their supports for the childbirth experience. Knowing how the nurses in the setting enact support will assist families in planning prenatally for the support needed during the childbirth event.

Administrative review is encouraged to assess the provisions made for the convoy in the way of a comfortable environment during their extended stay. It may be that the supports were observed getting up and down frequently not because of their need to provide support but, in part at least, because of a need to remove themselves from uncomfortable furniture. It is recognized that designers of labor and delivery settings in general, including those used for this study, have been acutely sensitive to consumer input and have created homelike environments unlike any experienced in most other acute care environments. Ongoing assessment is needed to evaluate particular aspects of the environment to determine not only their comfort, but their promotion and facilitation of supportive behaviors that promote positive childbirth outcomes. 


\section{Limitations of the Study}

Because I was the sole observer, much of the main focus of observation from minute to minute was on either the person who was speaking or, if no speaking was taking place, the person or persons moving about the setting. Due to this, nonverbal transactions among those not involved in the "action" at the moment may have been missed. Additionally, due to the fact that I was positioned in one location in the room throughout most of the event, there were many times that my view was only the back of some of the participants. This precluded me from observing nonverbal communications not within my line of sight. Pragmatically, in future studies these problems may be alleviated and additionally provide opportunity for post hoc microanalysis of the interactions by simultaneously recording activities in the room by multiple strategically-placed video cameras (Lowenberg, 1994).

The periods of observation, of considerable length, posed a challenge for continuous, open observations in that after extended periods I caught my mind wandering to other things and I was distracted by the uncomfortable furniture, room temperature and lighting in which I was attempting to do "work". Although prepared for labors of long duration with a bag of supplies, much the same as the labor convoy members provided for themselves, capturing research data in this natural setting was a challenge in that the setting was not designed for extended work. The nursing staff worked 8 
or 12 hour shifts and were not present throughout the women's entire stay and convoy members were in and out of the room. These unique challenges to data collection, while satisfying aspects of the credibility component for qualitative studies regarding prolonged engagement and persistent observation, reinforce the need for further validation of the theory due to these concerns regarding data dependability.

As natural as the setting was, it was most unnatural for me, as an observer writing on paper continuously, to be present in the setting. Although it was explained to each convoy member at the outset that my role was strictly observational and otherwise non-participatory. I was repeatedly brought into transactions. I was cared for like a "real" member of the convoy, being offered food, light, blankets and even to hold the newborn. My professional advice and knowledge was sought regarding treatments and expectations. The convoy's behavior toward me as an observer, did however, fit into the notion of support as a team approach. Perhaps just by being present I was considered by them to be a part of the team, with the mutual goal of supporting the laboring woman through childbirth. Continually monitoring my own behavior so as not to encourage interaction was a challenge. It is not known to what extent my presence and behavior influenced the transactions observed and future ethnographic research might well be designed with this in mind and include a 
triangulation method to ascertain the extent of transactions enacted as a result of observation.

Regarding the sample, the women who self-selected to be a part of this study were all from mid-to-high economic backgrounds. They all seemed to have a relatively stable life with, as far as I could tell, no other major life crises going on at the time of delivery. These factors may have contributed to communication styles and patterns preestablished among the convoy. It may also account for part of the reason that no incidents of outright conflict were witnessed. Future work in this area should include participants from a wider range of socioeconomic status with attention to prenatal communication patterns within the convoy.

Finally, the greatest limitation lies in the lack of post hoc validation of findings with the convoy members themselves. While the findings seem logical and plausible to the researcher and peer reviewer, triangulation with the thoughts and views of the other participants would greatly enrich the value of the preliminary theoretical views as presented.

Recommendations for Further Study

In future research, based on the experience of conducting this study, it would be interesting to investigate three major areas; post hoc validation of themes with the convoy members; how pre-labor patterns of support among the convoy members differ, if at all, from those 
during the childbirth experience; and cultural variations of convoy member activities. The decision to exclude physicians from the convoys observed in this study, in retrospect, seems to be upheld by the limited numbers of transactions observed between the physicians and other members in the setting. Therefore, it would be recommended that that exclusion be a part of future studies, where appropriate. One area of research would be to extend the qualitative methodology to include extensive post-event triangulation of the data with convoy-member follow-up. With an experienced observer, those follow-up validations could be attempted within hours of the childbirth events and/or in a week or more after the event. That validation would serve to further establish the credibility of the findings and assist in the development of theories of social support surrounding the primiparous childbirth event.

A second area of research would involve examining prelabor patterns of social support among convoy members to determine the extent to which those patterns are carried through the childbirth experience. Because childbirth is a unique situation in which primiparous families have no practice or experience, it is expected that some situationspecific supportive behaviors, like wiping the woman's buttocks, would be taken on temporarily. What is not known is the extent to which larger role adaptations are undertaken during childbirth as compared to a convoy's prelabor pattern. For example, a routinely directive, outgoing 
expectant father may become passive, while a routinely passive mother-in-law may assume the directive, facilitator role. Determining the nuances of these changes and to what degree the convoy members, including the laboring woman, expected them to happen, will provide practicing nurses and childbirth educators with additional information to impart to childbearing families preparing to experience childbirth for the first time (Coffman, Levitt, \& Brown, 1994; Stolte, 1987).

Finally, because the observations done for this study were only with caucasian women, a third area of research needed is a description of convoy transactions among support convoys of varying cultures (Callister, 1992). Additionally, it would be interesting to document convoy transactions when a mix of cultures are represented at the same childbirth event (Sharts-Hopko, 1995). 


\section{References}

Anderson, T. (1996). Support in labour. Modern Midwife, Jan. , 7-11.

Ahmann, E. (1994). Family-centered care: Shifting orientation. Pediatric Nursing, 20(2), 113-117.

Baldus, W. A. (1989). Physical environment should promote individual self-esteem. Provider, 33-4.

Balin, J. (1988). The sacred dimensions of pregnancy and birth. Qualitative Sociology, 11, 275-301.

Berry, L. M. (1988). Realistic expectation of the labor coach. Journal of obstetric, Gynecologic, and Neonatal Nursing, $17(5), \quad 354-355$.

Bertsch, T. D., Nagashima-Whalen, L., Dykeman, S., Kennell, J. H, \& McGrath, S. (1990). Labor support by first-time fathers: Direct observations with a comparison to experienced doulas. Journal of Psychosomatic Obstetrics and Gynaecology, 11, 251-260.

Bond, M. L., Keen-Payne, R. \& Lucy, P. W. (1995). The ideal nurse for the relinquishing mother: Lessons from the labor room. MCN, 20, 156-161.

Bouchard, L. (1993). Patients' satisfaction with the physical environment of an oncology clinic. Journal of Psychological Oncology, 11(1), 55-67.

Boyd, C. J. (1989). Mothers and daughters: A discussion of theory and research. Journal of Marriage and the Family, 51, 291-301. 
Boyle, J. S. (1994). Styles of Ethnography. In J. Morse (Ed.), Critical issues in qualitative research methods. Thousand Oaks: Sage.

Bryanton, J., Eraser-Davey, H. \& Sullivan, P. (1994). Women's perceptions of nursing support during labor. Journal of Obstetric, Gynecologic, and Neonatal Nursing, $\underline{23}(8), 638-644$.

Brown, M. A. (1986). Social support, stress, and health: A comparison of expectant mothers and fathers. Nursing Research, 35(2), 72-76.

Butler, J., Abrams, B., Parker, J., Roberts, J. M., \& Laros, R. K. (1993). Supportive nurse-midwife care is associated with a reduced incidence of cesarean section. American Journal of Obstetrics and Gynecology, 168(5), $1407-1413$.

Callister, L. C. (1992). The meaning of the childbirth experience of the Mormon Woman. The Journal of Perinatal Education, 1(1), 50-57.

Callister, L. C. (1995a). Becoming a father. The Journal of Perinatal Education, $4(1), 1-8$.

Callister, L. C. (1995b). Cultural meanings of childbirth. Journal of Obstetric, Gynecologic, and Neonatal Nursing, 24 (4), 327-331. 
Campbell, J. C. (1991). Voices and paradigms: Perspectives on critical and feminist theory in nursing. Advances in Nursing Science, 13 (3), 1-15.

Chalmers, B. \& Meyer, D. (1994). Companionship in the perinatal period: A cross-cultural survey of women's experiences. Journal of Nurse-Midwifery, 39(4), 265-272.

Chapman, L. L. (1992). Expectant fathers' roles during labor and delivery. Journal of Obstetric, Gynecologic, and Neonatal Nursing, 21(2), 114-120.

Coffman, S., Levitt, M. J., \& Brown, L. (1994). Effects of clarification of support expectations in prenatal couples. Nursing Research, 43(2), 111-116.

Collins, B. A. (1986). The role of the nurse in labor and delivery as perceived by nurses and patients. Journal of Obstetric, Gynecologic, and Neonatal Nursing, 15(5), 412418 .

Cooper, B. A. \& Hasselkus, B. R. (1992). Independent living and the physical environment: Aspects that matter to residents. Canadian Journal of Occupational Therapy, 59(1), $6-15$.

Cronenwett, L. R. (1985a). Network structure, social support, and psychological outcomes of pregnancy. Nursing Research, 34 (2), 93-99.

Cronenwett, L. R. (1985b). Parental network, structure and perceived support after birth of first child. Nursing Research, 34(6), 347-352. 
De Anda, D. \& Becerra, R. M. (1984). Support networks for adolescent mothers. Social Casework: The Journal of Contemporary Social Work, 65(3), 172-181.

Fischer, L. R. (1981). Transitions in the motherdaughter relationship. Journal of Marriage and the Family, $\underline{43}(3), 613-622$.

Germain, C. P. (1993). Ethnography: The Method. In P. Munhall \& C. Boyd (Eds.), Nursing research: A qualitative perspective. New York: National League for Nursing Press.

Geertz, C. (1973). The interpretation of cultures: Selected essays. New York: Basic Books, Inc.

Giblin, P. T., Poland, M. L., \& Ager, J.W. (1990). Effects of social supports on attitudes, health behaviors and obtaining prenatal care. Journal of Community Health, $15(6), 357-368$.

Gilgun, J.F. (1992). Definitions, methodologies, and methods in qualitative family research. In J. Gilgun, $\mathrm{K}$. Daly, \& G. Handel (Eds.), Qualitative Methods in Family Research. Sage Publications.

Gulrajani, R. P. (1995). Physical environmental factors affecting patients' stress in the accident and emergency department. Accident and Emergency Nursing, 3, $22-27$. 
Harrison, M. J., Neufeld, A., \& Kushner, K. (1995). Women in transition: Access and barriers to social support. Journal of Advanced Nursing, 21 858-864.

Hedstrom, I. W., \& Newton, N. (1986). Touch in labor: A comparison of cultures and eras. Birth, 13(3), 181-186.

Heerwagen, J. H., Heubach, J. G., Montgomery, J., \& Weimer, W. C. (1995). Environmental design, work, and well being. AAOHN Journal, $43(9)$.

Hodnett, E. D. (1996). Nursing support of the laboring woman. Journal of Obstetric, Gynecologic, and Neonatal Nursing, $25(3), 257-264$.

Hodnett, E. D., \& Osborn, R. W. (1989a). Effects of continuous intrapartum professional support on childbirth outcomes. Research in Nursing and Health, 12, 289-297.

Hodnett, E. D., \& Osborn, R. W. (1989b). A randomized trial of the effects of monitrice support during labor: Mothers' views two to four weeks postpartum. Birth, 16(4), $177-183$

Horman, S. (1989). The role of social support on health throughout the Iifecycle. Health Education, 18-21. Hupcey, J. E. \& Morse, J. M. (1997). Can a professional relationship be considered social support? Nursing Outlook, $45(6), 270-276$. 
Hutchinson, S. A. (1993). Grounded theory: The method. In P. Munhall \& C. Boyd (Eds.), Nursing Research: A Qualitative Perspective. N.Y.: National League for Nursing Press.

Hutton, J.D., \& Richardson, L. D. (1995). Healthscapes: The role of the Eacility and physical environment on consumer attitudes, satisfaction, quality assessments, and behaviors. Health Care Management Review, $\underline{20}(2), 48-61$.

Kahn, R. L., \& Antonucci, T. C. (1980). Convoys over the life course: Attachment, roles, and social support. In P. Baltes \& O. Brim (Eds.), Life-Span Development and Behavior (Vol. 3 ) (pp. 253-286). Academic Press.

Kennell, J., Klaus, M., McGrath, S., Robertson, S., \& Hinkley, C., (1991). Continuous emotional support during labor in a US hospital. Journal of the American Medical Association, 265 (17), 2197-2201.

King, C. R. (1990). The women's experience of childbirth on the western frontier. Journal of the West, $\underline{29}(1), 76-84$.

Kintz, D. L. (1987). Nursing support in labor. Journal of Obstetric, Gynecologic, and Neonatal Nursing, $16(2), \quad 126-130$.

Klass, S. S. (1988). A transcendent moment. MS. $\underline{16}(11), 40-41$. 
Klaus, M. H., Kennell, J. H., Robertson, S. S., \& Sosa, R. (1986). Effects of social support during parturition on maternal and infant morbidity. British Medical Journal, 293, $585-587$.

Klaus, M. H., Kennell, J. H., \& Klaus, P. H. (1993). Mothering the mother: How a doula can help you have a shorter, easier, and healthier birth. New York: AddisonWesley Publishing Company.

Labor of love: Doula care helps new moms. (1997, January). Women's Health Center Management, 11-13.

LaRossa, R. (1983). The transition to parenthood and the social reality of time. Journal of Marriage and the Family, 45 (3), 579-589.

Leininger, M. M. (1985). Ethnography and ethnonursing: Models and modes of qualitative data analysis. In M. Leininger (Ed.), Qualitative Research Methods in Nursing, Orlando: Grune \& Stratton, Inc.

Lincoln, Y. S., \& Guba, E. G. (1986). But is it rigorous? Trustworthiness and authenticity in naturalistic evaluation. In D. Williams (Ed.), Naturalistic Evaluation, San Francisco: Jossey-Bass.

Lindsey, A. M. (1988). Social support: Conceptualizations and measurement instruments. In M. FrankStromborg (Ed.), Instruments for Clinical Nursing Research Norwalk, Conn: Appleton \& Lange. 
Lofland, J. \& Lofland, I. H. (1984). Analyzing social settings. (2nd ed.). California: Wadsworth Publishing Company.

Lofland, J. \& Lofland, L. H. (1995). Analyzing social settings. (3rd ed.). California: Wadsworth Publishing Company.

Lothian, J. A. (1994). Is adequate labor support possible? The Journal of Perinatal Education, 3 (1), 40-41.

Lowenberg, J. S. (1993). Interpretive research methodology: Broadening the dialogue. Advances in Nursing Science, 16(2), 57-69.

Lowenberg, J. S. (1994). The nurse-patient relationship reconsidered: An expanded research agenda. Scholarly Inquiry for Nursing Practice: An International Journal, 8(2), 167-184.

Mackey, M. C., \& Lock, S. E. (1989). Women's expectations of the labor and delivery nurse. Journal of Obstetric, Gynecologic, and Neonatal Nursing, 18(6), 505512 .

Mackey, M. C. \& Stepans, M. E. (1994). Women's evaluations of their labor and delivery nurses. Journal of Obstetric, Gynecologic, and Neonatal Nursing, 23(5), 413420.

Malestic, S. L. (1990). Fathers need help during labor, too. $\mathrm{RN}, 53(7), 23-24$. 
Marshall, C., \& Rossman, G. B. (1989). Designing qualitative research. Newbury Park, CA: Sage.

Martell, L. K. (1990a). The mother-daughter relationship during daughter's first pregnancy: The transition experience. Holistic Nursing Practice, 4(3), $47-55$.

Martell, I. K. (1990b) . Perceptions of equity by mothers and daughters during daughters' first pregnancy. Family Relations, 39, 305-310.

Mattson, S. (1995). Culturally sensitive perinatal care for Southeast Asians. Journal of Obstetric, Gynecologic, and Neonatal Nursing, 24(4), 335-341.

McNiven, P., Hodnett, E., \& O'Brien-Pallas, L. L. (1992). Supporting women in labor: A work sampling study of the activities of labor and delivery nurses. Birth, $19(1), 3-8$.

Mercer, R. T., Nichols, E. G., \& Doyle, D. C. (1988). Transitions over the life cycle: A comparison of mothers and nonmothers. Nursing Research, 37(3), 144-151.

Miles, M. B., \& Huberman, A. M. (1994). Qualitative data analysis. (2nd Ed.). Thousand Oaks: Sage. Munhall, P. L. (1988). Ethical considerations in qualitative research. Western Journal of Nursing Research,10(2), 150-162. 
Munhall, P. L. (1993). Epistemology in nursing. In P. Munhall \& C. Boyd (Eds.), Nursing Research: A Qualitative Perspective. New York: National League for Nursing Press.

Murphy, S. A. (1990). Human responses to transitions: A holistic nursing perspective. Holistic Nursing Practice, $\underline{4}(3), 1-7$.

Namazi, K. H, \& Johnson, B. D. (1996). Issues related to behavior and the physical environment: Bathing cognitively impaired patients. Geriatric Nursing, 17(5), $234-239$.

Nichols, F. (1996). The meaning of the childbirth experience: A review of the literature. The Journal of Perinatal Education, 5(4), 71-77.

Nichols, M. R. (1993). Paternal perspectives of the childbirth experience. Maternal-Child Nursing Journal, $\underline{21}(3), \quad 99-108$.

Norbeck, J.S. (1981). Social support: A model for clinical research and application. Advances in Nursing Science, $3(4), 43$.

Norbeck, J. S. \& Tilden, V. P. (1983). Life stress, social support, and emotional disequilibrium in complications of pregnancy: A prospective, multivariate study. Journal of Health and Social Behavior, 24, 30-46. 
Patsdaughter, C. A., \& Killien, M. (1990).

Developmental transitions in adulthood: Mother-daughter relationships. Holistic Nursing Practice, 4(3), 37-46.

Purkis, M. E. (1996). Nursing in quality space:

Technologies governing experiences of care. Nursing Inquiry, 3, 101-111.

Raymond, T. (1992). Moms younger than eighteen and older than thirty-five: Should they have their own classes? International Journal of Childbirth Education, 7(4), 37-38.

Reece, S. M. (1993). Social support and the early maternal experience of primiparas over 35. Maternal-Child Nursing Journal, 21(3), 91-98.

Rosen, M. G. (1991). Doula at the bedside of the patient in labor. Journal of the American Medical Association, 265 (17), 2236-2237.

Saks, N. P. (1990). Utilizing an Ethnographic Approach to Survey a Labor and Delivery Waiting Room. Unpublished manuscript, University of San Diego.

Sandelowski. M. (1995). Sample size in qualitative research. Research in Nursing \& Health, 18, 179-183.

Sawyer, L., Regev, H., Proctor, S., Nelson, M., Messias, S., Barnes, D. \& Meleis, A. I. (1995). Matching versus cultural competence in research: Methodological considerations. Research in Nursing \& Health, 18, 557-567. 
Schatzman, L., \& Strauss, A. I. (1973). Field research: Strategies for a natural sociology. New Jersey: Prentice-Ha11, Inc.

Sharts-Hopko, N. C. (1995). Birth in the Japanese Context. Journal of Obstetric, Gynecologic, and Neonatal Nursing, 24(4), 343-351.

Shearer, B. (1984). Whose birth, whose body, whose baby is it? Birth, 11(3), 174-5.

Shearer, E. L., Shiono, P. H., \& Rhoads, G. G. (1988). Recent trends in family-centered maternity care for cesarean-birth families. Birth, 15(1), 3-7.

Sosa, R., Kennell, J., Klaus, M., Robertson, S., \& Urrutia, J. (1980). The effect of a supportive companion on perinatal problems, length of labor, and mother-infant interaction. The New England Journal of Medicine, 303 (11), $597-600$.

Stephany, T. (1983). Supporting the mother of a patient in labor. Journal of Obstetric, Gynecologic, and Neonatal Nursing, $12(5), 345-346$.

Stolte, K. (1987). A comparison of women's expectations of labor with the actual event. Birth, 14(2), $99-103$.

Stolte, K., Myers, S. T., \& Owen, W. L. (1994). Changes in maternity care and the impact on nurses and nursing practice. Journal of Obstetric, Gynecologic, and Neonatal Nursing, 23(7), 603-608. 
Tarkka, M. T. \& Paunonen, M. (1996). Social support and its impact on mothers' experiences of childbirth. Journal of Advanced Nursing, 23, 70-75.

Waldenstrom, U. (1996). Modern maternity care: Does safety have to take the meaning out of birth? Midwifery, 12, $165-173$.

Walker, A. J., \& Thompson, I. (1983). Intimacy and intergenerational aid and contact among mothers and daughters. Journal of Marriage and the Family, 45 (4), 841-9 Westreich, R., Spector-Dunsky, L., Klein, M., Papageorgiou, A., Kramer, M. \& Gelfand, M. (1991). The influence of birth setting on the father's behavior toward his partner and infant. Birth, 18(4), 198-202.

Williams, M. A. (1989). Physical environment of the intensive care unit and elderly patients. Critical care Nursing Quarterly, $12(1), 52-60$.

Young, D. (1992). Family-centered maternity care: Is the central nursery obsolete? Birth, 19(4), 183-184. 


\section{APPENDIX B}

Invitation to Participate

My name is Nancy Saks and I am currently a doctoral student at the University of San Diego School of Nursing. I am working on completing my dissertation which requires me to complete a program of research.

I am looking for woman and their supports who are interested in collaborating with me in the study of events of labor and childbirth. Participation in this study would require the following:

1) notification of Ms. Saks of the family's admission

to the Labor and Delivery area

2) permission for Ms. Saks to be present in the labor, delivery, recovery room (LDR) throughout the labor and childbirth and up to 1 hour after childbirth

3) permission from the woman and any individuals planning to be in the labor and childbirth room throughout the majority of the event

Participants in this research project can expect the satisfaction of knowing they have assisted in the expansion of nursing knowledge of the events of labor and delivery. A copy of the final written report will be made available to all participants.

If you are interested in participating, please answer the questions below and return this form to your childbirth education instructor. Thank you very much for considering participation in this research effort.

Name

Adaress

Home phone number

$\overline{\text { Mother's ethnicity }}$

Primary language
Due Date

Hospital

Number of individuals you are planning on having with you during your entire labor and childbirth at the hospital? 


\section{APPENDIX C \\ Consent to Participate}

I give permission for Nancy P. Saks, a doctoral candidate at the University of San Diego School of Nursing to include me in her study of labor and childbirth. I understand that data obtained from this research study will be used to increase nursing knowledge related to the care of families in the childbearing period.

I understand that my participation is completely voluntary and that I may withdraw from the study at any time without fear of consequence to myself or my family members.

There have been no arrangements made, financial or other, for me to be compensated for participation in this study.

I understand that at any time during the study that I have questions I can reach Ms. Saks at 578-0230 or 493-4028.

I may also contact her Dissertation Committee Chair, Dr. Colette Jones, at 260-4562.

I understand that my participation in this study is not expected to cause me physical, social, or psychological harm. A summary of the final findings will be made available to me by the researcher if I desire.

I understand, also, that my participation in this study grants Ms. Saks my permission to be present to observe and take notes throughout my labor and childbirth.

I understand that information obtained during this study will be kept confidential by the researcher. Subjects will remain anonymous in the final written report. Fictitious names or the grouping of data will be used.

No agreement has been made between myself and the researcher, written or verbal, beyond that expressed on this consent form. I have received a copy of this consent form.

I, the undersigned, understand the above explanations and, on that basis, I give consent to my voluntary participation in this research.

Signature of Subject

Date 
APPENDIX D

Sample Fieldnote Worksheet

Date

Page

Family Code

Time/progress

in labor

of

\%

\%

8

8

\%

\%

के

\%

g

\%

$\%$

\%

$\%$

8

\%

8

8

\%

$q$

\%

\%

$\%$

\%

q

$\frac{8}{8}$

8

$\frac{8}{8}$

$\frac{9}{8}$

$\frac{8}{8}$

8

\%

8

8

q

\%
Reflections

Observations

\%

8

q

8

के

q

q

q

q

웅

q

8

\%

o

8

\%

8

\%

\%

q

웅

웅

\%

के

8

各

웅

8

8

8

8

8

8

8

8

8 
APPENDIX E

Demographic Profile

Code

This sheet will be used by a variety of individuals.

For each area please circle the one response that is most appropriate in describing yourself.

$\begin{array}{lllll}\text { Sex } & \text { Age } & & \\ \text { 01. Male } & 01 . & \text { Under } 18 & 11 . & 55-58 \\ \text { 02. Female } & 02 . & 19-22 & 12 . & 59-62 \\ & 03 \cdot & 23-26 & 13 \cdot & 63-66 \\ & 04 . & 27-30 & 14 . & 67-70 \\ & 05 . & 31-34 & 15 . & 71+ \\ & 06 . & 35-39 & & \\ & 07 . & 40-42 & & \\ & 08 . & 43-46 & & \\ & 09 . & 47-50 & & \end{array}$

Relationship to laboring woman

01. Self

02. Spouse

03. Male friend or partner

04. Female friend

05. Sister

06. Aunt

07. Female cousin

08. Mother

09. Grandmother

10. Eemale nurse

11. Male nurse

12. Trained labor assistant

13. Non-RN staff member

14. Nursing student

15. Other (specify)

Attendance at Prenatal Classes?

01. Yes

02. No 